\title{
Electromagnetic spectral function and dilepton rate in a hot magnetized QCD medium
}

\author{
Snigdha Ghosh" and Vinod Chandra ${ }^{\dagger}$ \\ Indian Institute of Technology Gandhinagar, Palaj, Gandhinagar 382355, Gujarat, India
}

(Received 16 August 2018; published 9 October 2018)

\begin{abstract}
The dilepton production rate in a hot QCD medium is studied within an effective description of the medium in the presence of a magnetic field. This could be done by obtaining the one-loop self-energy of the photon due to the effective (quasi)quark loop at a finite temperature under an arbitrary external magnetic field while employing the real time formalism of thermal field theory. The effective quarks and gluons encode a hot QCD medium effective in terms of their respective effective fugacities. The magnetic field enters in the form of Landau level quantization, in the matter sector (quarks, antiquarks). The full Schwinger proper time propagator including all the Landau levels is considered for the quasiquarks while calculating the photon self-energy. The electromagnetic Debye screening (in terms of the self-energy) has been influenced by both the hot QCD medium effects and the magnetic field. Analogous results are also obtained from the semiclassical transport theory. The imaginary part of the photon self-energy function is obtained from the discontinuities of the self-energy across the unitary cuts that are also present at the zero magnetic field and the Landau cuts that are purely due to the magnetic field. The dilepton production rate is then obtained in terms of the product of electromagnetic spectral functions due to the quark loop and the lepton loop. The modifications of both the quarks/antiquarks as well as leptons in the presence of an arbitrary external magnetic field have been considered in the formalism. Significant enhancement of the low invariant mass dileptons due to the appearance of the Landau cuts in the electromagnetic spectral function at the finite external magnetic field has been observed. A substantial enhancement of the dilepton rate is also found when the equation of state effects are considered through the effective quarks/antiquraks.
\end{abstract}

DOI: 10.1103/PhysRevD.98.076006

\section{INTRODUCTION}

The study of the nuclear matter under extreme conditions of temperature and/or density has been a subject of intense investigation over the past few decades. Heavy ion collision (HIC) experiments in the Relativistic Heavy Ion Collider (RHIC) and Large Hadron Collider (LHC) are expected to produce such a state in the laboratory where effectively free quarks and gluons become nearly thermalized over the nuclear volume scale. This form of the nuclear matter is commonly termed as quark-gluon plasma (QGP), which is more like a near perfect [1-4] and most vortical fluid [5] that could be created in the experiments. The experimental observations strongly indicate toward the strongly interacting nature of the QGP. Recent studies have further revealed that, in a noncentral or asymmetric HIC, extremely high

\footnotetext{
*snigdha.physics@gmail.com, snigdha.ghosh@iitgn.ac.in vchandra@iitgn.ac.in
}

Published by the American Physical Society under the terms of the Creative Commons Attribution 4.0 International license. Further distribution of this work must maintain attribution to the author(s) and the published article's title, journal citation, and DOI. Funded by SCOAP ${ }^{3}$. magnetic fields are created [6]. The magnitude of the magnetic field is comparable to the typical QCD energy scale $\left(e B \sim \Lambda_{\mathrm{QCD}}^{2}\right)$, and thus it could nontrivially affect bulk as well microscopic properties of the QGP. In fact, the strength of the magnetic field turned out to be larger than fields present in the interior of a magnetar [7] though in the very early stages of the HIC.

Next, the study of this strongly interacting matter at high temperature and density along with an external magnetic field has gained extreme momentum over the past decade [8] because of many exotic effects such as the chiral magnetic effect (CME), magnetic catalysis (MC), inverse magnetic catalysis (IMC), the chiral vortical effect (CVE), and the superconductivity of the vacuum [9-16] that can happen when the hot and dense QCD matter is placed under a high external magnetic field. Notably, these studies are also important in the context of astrophysics and cosmology [7,17-23].

The QGP formed in a HIC experiment is a "transient" state, which exists for a very short time $(\sim$ few fm/c) implying that it cannot be observed directly (the direct detection is also prohibited by the color confinement). There are various indirect probes and observables [24] that are used to extract the properties of this hot and dense 
medium such as electromagnetic probes (photon and dileptons) [25-34], heavy quarks [35], quarkonia [36], jets [37], and collective flow [1,38-42]. Another important theoretical tool to probe the microscopic properties of the medium has been the study of different $n$-point currentcurrent correlation functions at finite temperature and density (the in-medium spectral functions of local currents). The transport quantities such as shear and bulk viscosities and thermal conductivity could be obtained from the spectral functions consisting of appropriate currents. In our context, the prime interest is in the electromagnetic spectral function, which is obtained from the vector-vector current correlator. The correlator can also be related to the dilepton production rate (DPR) from the hot and dense magnetized QGP medium. The dileptons are emitted from the entire spacetime volume throughout the medium evolution. Because of the fact that the dileptons interact only through the electromagnetic interaction, they have larger mean free paths. The dileptons come out of the thermal medium soon after their production without suffering more collisions. Thus the dileptons carry the precise information of the thermodynamic state of the medium where they are produced. In the QGP medium, a quark interacts with an antiquark to produce a virtual photon that subsequently decays into a dilepton. In addition to this, there are other sources of dileptons in a HIC experiment such as the interaction of charged hadrons with their antiparticles like $\pi^{+} \pi^{-} \rightarrow l^{+} l^{-}$that can produce the dileptons. They can also be produced from the decays of hadron resonances $\left(\pi^{0}, \rho, \omega, J / \psi\right)$. The Drell-Yan process also gives a significant contribution to the high invariant mass dilepton productions.

In order to calculate the electromagnetic spectral function and DPR, the most essential input is the local equilibrium distribution functions of the quarks/antiquarks that describes the interacting hot QCD/QGP medium. At this juncture, a recently developed effective fugacity quasiparticle model (EQPM) [43] provides a systematic description of a hot QCD medium in accordance with the realistic QCD equation of state (EOS) in terms of effective temperature dependent fugacities in the thermal distribution of gluons and quarks/ antiquarks that encode all the effects of the strong interaction. The temperature dependences of the effective fugacities could be obtained from the realistic hot QCD equations of state such as recent lattice QCD EOS.

The photon polarization tensor under the external magnetic field has been calculated earlier at zero temperature in Refs. [44-47] and also at finite temperature in Ref. [48]. The DPR in the presence of the external magnetic field has been studied earlier in Refs. [49-54]. In Refs. [49,50], the authors have obtained the DPR from hot magnetized QGP in a phenomenological way including the effects of synchrotron radiation as well as quark-antiquark annihilation, whereas in Ref. [51], the authors have used the Ritus formalism to calculate the photon polarization tensor and
DPR under the external magnetic field. Recently the DPR in strong as well as in weak magnetic field approximation has been obtained in Refs. [53,54]. In all of the mentioned works, the authors have considered the ideal Fermi-Dirac distribution function for the quarks/antiquarks for the calculation of DPR. In contrast, in Ref. [55], the DPR has been obtained using the modified equilibrium distribution functions of quarks/antiquarks within the EQPM at the zero external magnetic field where the effects of viscous modification are also included. This sets the motivation for the present work, where we aim for a systematic inclusion of magnetic field effects and realistic QGP equation of state in the field theoretical formalism.

In this work, we aim to calculate the photon polarization tensor with the effective quarks as the loop particles (incorporating EQPM) in the presence of the arbitrary external magnetic field at finite temperature. The real time formalism (RTF) of thermal field theory (TFT) has been employed for the calculation of one-loop photon selfenergy at finite temperature. The full Schwinger proper time propagator including all the Landau levels for the quasiquark propagation is considered in the calculation. No strong or weak field approximation has been made in the analysis as is usually done in most of the works in the literature. The Debye screening mass for the electromagnetic screening in the hot magnetized QCD medium is obtained by taking the static limit of the 00 component of the photon polarization tensor $\left(\Pi^{\mu \nu}\right)$, and it matches the one obtained from the semiclassical transport theory. Further, the imaginary part of the self-energy is obtained from the discontinuities across the unitary and Landau cuts. For physical timelike momentum of the external photon, the unitary as well as the Landau cuts are observed. The unitary cuts are also present at zero temperature and zero external magnetic field, whereas the Landau cuts only appear at finite temperature and only when the loop particles have different masses. In our case, although the loop particles are both quarks having equal mass, the Landau cuts appear due to the external magnetic field. The appearance of the Landau cuts have correspondence to the physical processes such as photon emission or absorption by a quark/antiquark in magnetized QGP, which was forbidden in the zero magnetic field case due to kinematic restrictions. The analytic structure of the imaginary part of the self-energy has also been analyzed, and we found that the thresholds of the unitary and Landau cuts get modified due to the presence of the external magnetic field. Finally, the DPR is obtained from the thermal QGP medium while expressing it in terms of the electromagnetic spectral functions due to the quark loop and the lepton loop.

Note that there has been another method used in the literature for obtaining the leptonic part in DPR by taking into account the spin sums over the leptonic spinor $[53,56]$. We have shown explicitly that these two different approaches converge to the same result. However, this 
particular form of the DPR in terms of the spectral function due to the quark and lepton loops enables us to introduce the external magnetic field through the modification of the quark and lepton propagators in terms of Schwinger proper time one where a knowledge of the spin sum over the leptonic spinor in the presence of the external magnetic field is not required. We have found a significant impact on the low invariant mass dilepton yield due to the external magnetic field. The DPR also shows significant enhancement when the QCD medium effects are considered via the EQPM.

The paper is organized as follows. In Sec. II, we have briefly discussed the EQPM. In Sec. III, the one-loop photon self-energy due to the quark loop is obtained at finite temperature in the absence of the external magnetic field. This is followed by the calculation of the photon polarization tensor under the external magnetic field in addition to the finite temperature in Sec. IV. After that, we have discussed the Debye screening mass in Sec. V and the electromagnetic spectral functional along with its analytic structure in Sec. VI. Sections VII and VIII are devoted to the calculation of DPR at the zero and finite external magnetic fields, respectively. The numerical results are provided in Sec. IX, and finally we summarize and conclude in Sec. X. Some of the relevant calculational details are provided in the Appendices.

\section{THE EQPM}

The EQPM describes hot QCD medium effects that are encoded in the realistic hot QCD EOSs in terms of a grand canonical system of effective quarks, antiquarks, and gluons. In this scheme, the hot QCD medium effects encoded in the realistic hot QCD EOSs (either computed from lattice QCD or Hard Thermal Loop perturbation theory) could be introduced in terms of effective quark/ antiquark and effective gluon distribution functions. In this work, the EQPM developed in Ref. [43] and later generalized for the magnetized hot QCD medium in Ref. [57] is utilized to include hot magnetized QCD medium effects. The EQPM maps the hot QCD EOSs in terms of effective quark-antiquarks and effective gluons with effective fugacities. The effective fugacities in the model are merely introduced to capture the hot QCD medium effects and could be understood in terms of nontrivial dispersion for effective quarks and gluons. They should not be confused with the presence of any conserved current. Their physical meaning is reflected in the modified dispersion of quasiparticles and in terms of the mean field contribution in the effective covariant kinetic theory [58].

Notably, there are other effective quasiparticle models to describe the hot QCD medium effects with effective masses for quarks and gluons [59,60], effective masses with the Polyakov loop [61-64], Nambu-Jona-Lasinio and Polyakov Nambu-Jona-Lasinio based quasiparticle models [65-69], and self-consistent and single parameter quasiparticle models [70-72]. There are a few other recently proposed quasiparticle models based on the Gribov-Zwanziger (GZ) quantization [73-76]. All these models tried to interpret interacting hot QCD medium in terms of noninteracting/weakly interacting quasiparticles with some effective parameters that capture all the medium effects.

The EQPM describes the quasigluon and quasiquark/ antiquark in terms of their respective distribution functions

$\tilde{f}(|\vec{k}|)=\frac{1}{z_{g}^{-1} e^{\beta|\vec{k}|}-1} \quad$ and $\quad f\left(\omega_{k}^{f}\right)=\frac{1}{z_{q}^{-1} e^{\beta \omega_{k}^{f}}+1}$,

where $\omega_{k}^{f}=\sqrt{\vec{p}^{2}+m_{f}^{2}}$ with $m_{f}$ being the mass of quarks/ antiquark flavor $f$. The physical significance of the effective fugacities $z_{g, q}$ comes in the dispersion relations of gluons and quarks as

$\tilde{\omega}_{k}^{g}=|\vec{k}|+T^{2} \frac{\partial}{\partial T} \ln \left(z_{g}\right) \quad$ and $\quad \tilde{\omega}_{k}^{f}=\omega_{k}^{f}+T^{2} \frac{\partial}{\partial T} \ln \left(z_{q}\right)$.

Both $z_{g}$ and $z_{q}$ have complicated temperature dependence as discussed in Ref. [77]. Here, we consider the EQPM description of the recent $(2+1)$ flavor lattice QCD EOS $[78,79]$. Notably, the magnitudes of $z_{g}$ and $z_{q}$ are always less than unity. The asymptotic limit, $z_{q}=z_{g}=1$, is equivalent to ideal EOS (Stefan-Boltzmann limit) for the QCD. The fugacities, $z_{g}=z_{g}(T)<1$ and $z_{q}=z_{q}(T)<1$, have been constructed in such a way that a grand canonical ensemble of the noninteracting quasipartons leads to an effective EOS identical to the Lattice QCD (LQCD) one. In this work we have presented the numerical results of all the quantities for two different cases separately: (i) $z_{q}=z_{g}=1$ abbreviated as "ideal EOS" and (ii) $z_{q}=z_{q}(T)<1, z_{g}=z_{g}(T)<1$ abbreviated as "LQCD EOS." The extension of EQPM in the magnetic field background involves the modification of dispersion relation by relativistic Landau levels as discussed in [57]. Extended EQPM for hot magnetized QGP has further been employed to study the transport coefficients of medium [80,81] while employing the covariant kinetic theory developed in the context of EQPM in Ref. [58].

\section{PHOTON SELF-ENERGY AT FINITE TEMPERATURE}

The Lagrangian for $\gamma q \bar{q}$ electromagnetic interaction is given by

$$
\mathcal{L}_{\mathrm{int}}=\sum_{f} e_{f} \bar{q}_{f} \gamma^{\mu} q_{f} A_{\mu},
$$

where $q_{f}\left(\bar{q}_{f}\right)$ is the quark(antiquark) field, $A_{\mu}$ is the photon field, and $e_{f}=\sqrt{4 \pi \alpha} Q_{f}$ is the electric charge for quark 
flavor $f$. Here $Q_{f}=\frac{2}{3}$ for $f \equiv(u, c, t)$ and $Q_{f}=-\frac{1}{3}$ for $f \equiv(d, s, b) . \alpha=\frac{1}{137}$ is the QED fine structure constant. Using Eq. (3), the one-loop vacuum self-energy of the photon can be written as (by applying the Feynman rule to Fig. 1)

$$
\Pi_{\mathrm{vac}}^{\mu \nu}(q)=-\sum_{f} e_{f}^{2} N_{c} i \int \frac{d^{4} k}{(2 \pi)^{4}} \operatorname{Tr}\left[\gamma^{\nu} S_{F}^{f}(p=q+k) \gamma^{\mu} S_{F}^{f}(k)\right],
$$

where $N_{c}$ is the number of color and

$$
S_{F}^{f}(p)=\frac{-\left(\not p+m_{f}\right)}{p^{2}-m_{f}^{2}+i \epsilon}
$$

is the vacuum Feynman propagator for a spin $-\frac{1}{2}$ particle.

In order to calculate the photon self-energy at the finite temperature, we use the standard techniques of RTF of TFT in which all the two point correlation functions such as propagator and self-energy become $2 \times 2$ matrices in thermal indices [56,82]. However, they can be put in diagonal forms in terms of analytic functions that we will denote by a bar. This also enables one to express the analytic function in terms of any one component of the $2 \times 2$ matrix, say the 11 component. Denoting the 11 component of the thermal photon self-energy matrix by $\Pi_{11}^{\mu \nu}\left(q^{0}, \vec{q}\right)$, we can write

$\Pi_{11}^{\mu \nu}\left(q^{0}, \vec{q}\right)=-\sum_{f} e_{f}^{2} N_{c} i \int \frac{d^{4} k}{(2 \pi)^{4}} \operatorname{Tr}\left[\gamma^{\nu} S_{11}^{f}(p) \gamma^{\mu} S_{11}^{f}(k)\right]$,

where $S_{11}^{f}(p)$ is the 11 component of the real time Dirac propagator given by

$$
S_{11}^{f}(p)=S_{F}^{f}(p)-\eta(p \cdot u)\left[S_{F}^{f}(p)-\gamma^{0} S_{F}^{f^{\dagger}}(p) \gamma^{0}\right] .
$$

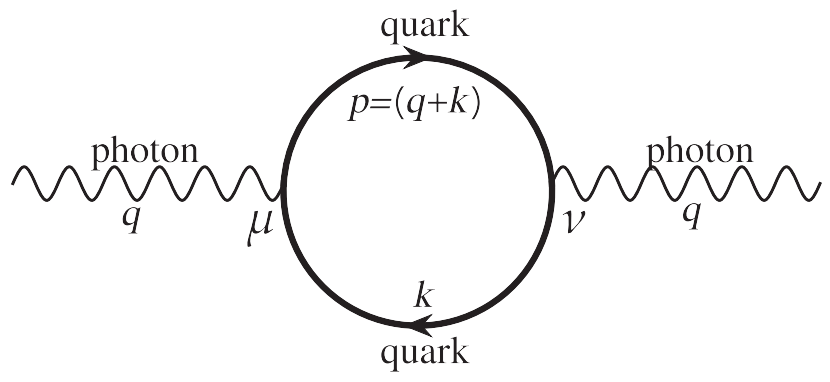

FIG. 1. Feynman diagram for one-loop photon self-energy.

In the above equation, $\eta(p \cdot u)=\Theta(p \cdot u) f(p \cdot u)+$ $\Theta(-p \cdot u) f(-p \cdot u)$ with the quasiquark thermal distribution function $f$ defined in Eq. (1). Here $u^{\mu}$ is the four velocity of the thermal medium which in the local rest frame reduces to $u^{\mu} \equiv(1, \overrightarrow{0}), \beta$ is the inverse temperature, and $\Theta(x)$ is the unit step function. It is to be noted that the quark distribution function in the thermal propagator contains the effective fugacity parameter $z_{q}=z_{q}(T)$. As we already mentioned, $\Pi_{11}^{\mu \nu}\left(q^{0}, \vec{q}\right)$ is related to the analytic thermal self-energy function of photon by the following relations $[56,82]$

$$
\operatorname{Re} \bar{\Pi}^{\mu \nu}\left(q^{0}, \vec{q}\right)=\operatorname{Re} \Pi_{11}^{\mu \nu}\left(q^{0}, \vec{q}\right),
$$

$\operatorname{Im} \bar{\Pi}^{\mu \nu}\left(q^{0}, \vec{q}\right)=\operatorname{sign}\left(q^{0}\right) \tanh \left(\frac{\beta q^{0}}{2}\right) \operatorname{Im} \Pi_{11}^{\mu \nu}\left(q^{0}, \vec{q}\right)$,

where $\operatorname{sign}(x)=\Theta(x)-\Theta(-x)$ is the signum function. We now rewrite Eq. (7) as

$$
\begin{aligned}
S_{11}^{f}(p)= & \left(\not p+m_{f}\right)\left[\left(\frac{-1}{p^{2}-m_{f}^{2}+i \epsilon}\right)\right. \\
& \left.-\eta(p \cdot u) 2 \pi i \delta\left(p^{2}-m_{f}^{2}\right)\right]
\end{aligned}
$$

and substitute into Eq. (6). Performing the $d k^{0}$ integration and using Eqs. (8) and (9) we obtain the real and imaginary parts of the photon self-energy function as

$$
\begin{aligned}
\operatorname{Re} \bar{\Pi}^{\mu \nu}\left(q^{0}, \vec{q}\right)= & \operatorname{Re} \Pi_{\text {vac }}^{\mu \nu}(q)+\sum_{f} e_{f}^{2} N_{c} \int \frac{d^{3} k}{(2 \pi)^{3}} \mathcal{P}\left[\frac{f\left(\omega_{k}^{f}\right)}{2 \omega_{k}^{f}}\left\{\frac{N_{f}^{\mu \nu}\left(k^{0}=-\omega_{k}^{f}\right)}{\left(q^{0}-\omega_{k}^{f}\right)^{2}-\left(\omega_{p}^{f}\right)^{2}}+\frac{N_{f}^{\mu \nu}\left(k^{0}=\omega_{k}^{f}\right)}{\left(q^{0}+\omega_{k}^{f}\right)^{2}-\left(\omega_{p}^{f}\right)^{2}}\right\}\right. \\
& \left.\times \frac{f\left(\omega_{p}^{f}\right)}{2 \omega_{p}^{f}}\left\{\frac{N_{f}^{\mu \nu}\left(k^{0}=-q^{0}-\omega_{p}^{f}\right)}{\left(q^{0}+\omega_{p}^{f}\right)^{2}-\left(\omega_{k}^{f}\right)^{2}}+\frac{N_{f}^{\mu \nu}\left(k^{0}=-q^{0}+\omega_{p}^{f}\right)}{\left(q^{0}-\omega_{p}^{f}\right)^{2}-\left(\omega_{k}^{f}\right)^{2}}\right\}\right],
\end{aligned}
$$

$$
\begin{aligned}
\operatorname{Im} \bar{\Pi}^{\mu \nu}\left(q^{0}, \vec{q}\right)= & \operatorname{sign}\left(q^{0}\right) \tanh \left(\frac{\beta q^{0}}{2}\right) \sum_{f} e_{f}^{2} N_{c} \pi \int \frac{d^{3} k}{(2 \pi)^{3}} \frac{1}{4 \omega_{k}^{f} \omega_{p}^{f}}\left[\left\{1-f\left(\omega_{k}^{f}\right)-f\left(\omega_{p}^{f}\right)+2 f\left(\omega_{k}^{f}\right) f\left(\omega_{p}^{f}\right)\right\}\right. \\
& \times\left\{N_{f}^{\mu \nu}\left(k^{0}=-\omega_{k}^{f}\right) \delta\left(q^{0}-\omega_{k}^{f}-\omega_{p}^{f}\right)+N_{f}^{\mu \nu}\left(k^{0}=\omega_{k}^{f}\right) \delta\left(q^{0}+\omega_{k}^{f}+\omega_{p}^{f}\right)\right\}+\left\{-f\left(\omega_{k}^{f}\right)-f\left(\omega_{p}^{f}\right)+2 f\left(\omega_{k}^{f}\right) f\left(\omega_{p}^{f}\right)\right\} \\
& \left.\times\left\{N_{f}^{\mu \nu}\left(k^{0}=-\omega_{k}^{f}\right) \delta\left(q^{0}-\omega_{k}^{f}+\omega_{p}^{f}\right)+N_{f}^{\mu \nu}\left(k^{0}=\omega_{k}^{f}\right) \delta\left(q^{0}+\omega_{k}^{f}-\omega_{p}^{f}\right)\right\}\right],
\end{aligned}
$$

where $\mathcal{P}$ denotes the Cauchy principal value and 


$$
N_{f}^{\mu \nu}(q, k)=\operatorname{Tr}\left[\gamma^{\nu}\left(\not 1+\not k+m_{f}\right) \gamma^{\mu}\left(\not k+m_{f}\right)\right]=4\left[\left(m_{f}^{2}-k^{2}-k \cdot q\right) g^{\mu \nu}+2 k^{\mu} k^{\nu}+\left(k^{\mu} q^{\nu}+k^{\nu} q^{\mu}\right)\right] .
$$

The first term on the right-hand side (RHS) of Eq. (11) is ultraviolet divergent. Performing dimensional regularization followed by the $\overline{\mathrm{MS}}$ scheme, we get [83]

$$
\operatorname{Re} \Pi_{\mathrm{vac}}^{\mu \nu}(q)=\left(q^{2} g^{\mu \nu}-q^{\mu} q^{\nu}\right) \sum_{f} \frac{e_{f}^{2} N_{c}}{2 \pi^{2}} \int_{0}^{1} d x x(x-1) \ln \left|\frac{m_{f}^{2}-x(1-x) q^{2}}{\Lambda}\right|,
$$

where $\Lambda$ is a scale of dimension $\mathrm{GeV}^{2}$. It is worth mentioning that only the pure vacuum contribution contains the ultraviolet divergence, whereas the temperature dependent part is free from any divergences [82]. An analogous argument will also hold for the self-energy in the presence of the external magnetic field where the divergent part will only come from the pure vacuum contribution, whereas the magnetic field dependent terms will be finite. However, the imaginary part of the self-energy is finite and scale independent. Thus the electromagnetic spectral function, as well as DPR, obtained from the imaginary part will also be independent of scale and regularization scheme. On the other hand, the contribution of the scale dependent pure vacuum self-energy to the Debye mass is zero, which will be discussed in Sec. V.

\section{PHOTON SELF-ENERGY AT FINITE TEMPERATURE UNDER EXTERNAL MAGNETIC FIELD}

In the presence of a constant external magnetic field $\vec{B}=B \hat{z}$, the 11 component of the quasiquark propagator becomes [48]

$$
S_{11}^{f}(p)=S_{B}^{f}(p)-\eta(p \cdot u)\left[S_{B}^{f}(p)-\gamma^{0} S_{B}^{f \dagger}(p) \gamma^{0}\right]
$$

where $S_{B}^{f}(p)$ is the momentum space Schwinger proper time propagator for a charged spin- $\frac{1}{2}$ particle given by $[84,85]$

$$
S_{B}^{f}(p)=i \int_{0}^{\infty} d s \exp \left[i s\left\{p_{\|}^{2}+\frac{\tan \left(e_{f} B s\right)}{e_{f} B s} p_{\perp}^{2}-m_{f}^{2}\right\}\right]\left[\left(\not \|_{\|}+m_{f}\right)\left\{1-\gamma^{1} \gamma^{2} \tan \left(e_{f} B s\right)\right\}+\not \perp_{\perp} \sec ^{2}\left(e_{f} B s\right)\right]
$$

Since we are considering the magnetic field along the $+v e z$ direction, we decompose the metric tensor $g^{\mu \nu}=\left(g_{\|}^{\mu \nu}+g_{\perp}^{\mu \nu}\right)$ where $g_{\|}^{\mu \nu}=\operatorname{diag}(1,0,0,-1)$ and $g_{\perp}^{\mu \nu}=\operatorname{diag}(0,-1,-1,0)$ so that $p_{\|}^{\mu}=p_{\nu} g_{\|}^{\mu \nu}$ and $p_{\perp}^{\mu}=p_{\nu} g_{\perp}^{\mu \nu}$. It is worth mentioning that the corresponding coordinate space Schwinger propagator contains a translationally noninvariant phase factor. It can be shown that, for one-loop self-energy graphs of neutral particles (such as the photon in our case) in which the loop particles are equally charged, the phase factor gets canceled. In our case, we can work with the momentum space quasiquark propagator. The proper time integral in Eq. (16) can be performed so that the propagator can be written as a sum over Landau levels as

$$
S_{B}^{f}(p)=-\sum_{n=0}^{\infty}\left[\frac{(-1)^{n} e^{-\alpha_{p}} D_{n}^{f}(p)}{p_{\|}^{2}-m_{f}^{2}-2 n\left|e_{f} B\right|+i \epsilon}\right]
$$

where $\alpha_{p}=-\frac{p_{\perp}^{2}}{\left|e_{f} B\right|}$ and

$$
D_{n}^{f}(p)=\left(\not \not_{\|}+m_{f}\right)\left[\left\{1+\operatorname{sign}\left(e_{f}\right) i \gamma^{1} \gamma^{2}\right\} L_{n}\left(2 \alpha_{p}\right)-\left\{1-\operatorname{sign}\left(e_{f}\right) i \gamma^{1} \gamma^{2}\right\} L_{n-1}\left(2 \alpha_{p}\right)\right]-4 \not{ }_{\perp} L_{n-1}^{1}\left(2 \alpha_{p}\right)
$$

in which $L_{n}^{a}(z)$ are the generalized Laguerre polynomial with $L_{-1}^{a}(z)=0$. We now rewrite Eq. (15) using Eq. (17) as

$$
S_{11}^{f}(p)=\sum_{n=0}^{\infty}(-1)^{n} e^{-\alpha_{p}} D_{n}^{f}(p)\left[\left(\frac{-1}{p_{\|}^{2}-m_{f}^{2}-2 n\left|e_{f} B\right|+i \epsilon}\right)-\eta(p \cdot u) 2 \pi i \delta\left(p_{\|}^{2}-m_{f}^{2}-2 n\left|e_{f} B\right|\right)\right],
$$

and substitute into Eq. (6). After performing the $d k^{0}$ integral and using Eqs. (8) and (9) we obtain the real and imaginary parts of the photon self-energy function at finite temperature under the external magnetic field as 


$$
\begin{aligned}
\operatorname{Re} \bar{\Pi}^{\mu \nu}\left(q^{0}, \vec{q}\right)= & \sum_{f} e_{f}^{2} N_{c} \sum_{l=0}^{\infty} \sum_{n=0}^{\infty} \int \frac{d^{3} k}{(2 \pi)^{3}} \mathcal{P}\left[\frac{f\left(\omega_{k, l}^{f}\right)}{2 \omega_{k, l}^{f}}\left\{\frac{N_{f, n l}^{\mu \nu}\left(k^{0}=-\omega_{k, l}^{f}\right)}{\left(q^{0}-\omega_{k, l}^{f}\right)^{2}-\left(\omega_{p, n}^{f}\right)^{2}}+\frac{N_{f, n l}^{\mu \nu}\left(k^{0}=\omega_{k, l}^{f}\right)}{\left(q^{0}+\omega_{k, l}^{f}\right)^{2}-\left(\omega_{p, n}^{f}\right)^{2}}\right\}\right. \\
& \left.\times \frac{f\left(\omega_{p, n}^{f}\right)}{2 \omega_{p, n}^{f}}\left\{\frac{N_{f, n l}^{\mu \nu}\left(k^{0}=-q^{0}-\omega_{p, n}^{f}\right)}{\left(q^{0}+\omega_{p, n}^{f}\right)^{2}-\left(\omega_{k, l}^{f}\right)^{2}}+\frac{N_{f, n l}^{\mu \nu}\left(k^{0}=-q^{0}+\omega_{p, n}^{f}\right)}{\left(q^{0}-\omega_{p, n}^{f}\right)^{2}-\left(\omega_{k, l}^{f}\right)^{2}}\right\}\right]+\operatorname{Re} \Pi_{v a c}^{\mu \nu}(q)+\operatorname{Re} \Pi_{\mathrm{B}}^{\mu \nu}(q, B),
\end{aligned}
$$

$$
\begin{aligned}
\operatorname{Im} \bar{\Pi}^{\mu \nu}\left(q^{0}, \vec{q}\right)= & \operatorname{sign}\left(q^{0}\right) \tanh \left(\frac{\beta q^{0}}{2}\right) \sum_{f} e_{f}^{2} N_{c} \sum_{l=0}^{\infty} \sum_{n=0}^{\infty} \pi \int \frac{d^{3} k}{(2 \pi)^{3}} \frac{1}{4 \omega_{k, l}^{f} \omega_{p, n}^{f}}\left[\left\{1-f\left(\omega_{k, l}^{f}\right)-f\left(\omega_{p, n}^{f}\right)+2 f\left(\omega_{k, l}^{f}\right) f\left(\omega_{p, n}^{f}\right)\right\}\right. \\
& \times\left\{N_{f, n l}^{\mu \nu}\left(k^{0}=-\omega_{k, l}^{f}\right) \delta\left(q^{0}-\omega_{k, l}^{f}-\omega_{p, n}^{f}\right)+N_{f, n l}^{\mu \nu}\left(k^{0}=\omega_{k, l}^{f}\right) \delta\left(q^{0}+\omega_{k, l}^{f}+\omega_{p, n}^{f}\right)\right\} \\
& +\left\{-f\left(\omega_{k, l}^{f}\right)-f\left(\omega_{p, n}^{f}\right)+2 f\left(\omega_{k, l}^{f}\right) f\left(\omega_{p, n}^{f}\right)\right\} \\
& \left.\times\left\{N_{f, n l}^{\mu \nu}\left(k^{0}=-\omega_{k, l}^{f}\right) \delta\left(q^{0}-\omega_{k, l}^{f}+\omega_{p, n}^{f}\right)+N_{f, n l}^{\mu \nu}\left(k^{0}=\omega_{k, l}^{f}\right) \delta\left(q^{0}+\omega_{k, l}^{f}-\omega_{p, n}^{f}\right)\right\}\right]
\end{aligned}
$$

where

$$
\begin{aligned}
& \omega_{k, l}^{f}=\sqrt{k_{z}^{2}+m_{f, l}^{2}} \text { with } m_{f, l}=\sqrt{m_{f}^{2}+2 n\left|e_{f} B\right|} \text { and } \\
& N_{f, n l}^{\mu \nu}(q, k)=(-1)^{n+l} e^{-\alpha_{k}-\alpha_{p}} \operatorname{Tr}\left[\gamma^{\nu} D_{n}^{f}(p) \gamma^{\mu} D_{l}^{f}(k)\right] .
\end{aligned}
$$

It is worth mentioning that the last term in Eq. (20), i.e., $\operatorname{Re} \Pi_{\mathrm{B}}^{\mu \nu}(q, B)$, is the magnetic field dependent vacuum contribution to the real part of the self-energy which is temperature independent. This term will contribute to the dispersion relations of the photon. A similar study incorporating this magnetic field dependent vacuum contribution term, for the dispersion relations of $\pi$ and $\rho$ mesons, can be found in Refs. [86-88]. However, in this work, we have not given the explicit calculation of this term since it does not contribute to the Debye mass, which we will discuss in the next section.

\section{DEBYE SCREENING MASS}

The Debye screening mass $m_{D}$ for electromagnetic screening can be obtained by taking the static limit of the 00 component of the photon polarization tensor,

$$
m_{D}^{2}=-\operatorname{Re} \bar{\Pi}^{00}\left(q^{0}=0, \vec{q} \rightarrow \overrightarrow{0}\right) .
$$

Let us first calculate $m_{D}$ for the zero magnetic field case. From Eq. (11) we get

$$
\begin{aligned}
\lim _{q^{0}} & =0, \vec{q} \rightarrow \overrightarrow{0} \\
& \operatorname{Re} \bar{\Pi}^{00}\left(q^{0}, \vec{q}\right) \\
& =-\left(\frac{4 N_{c}}{T}\right) \sum_{f} e_{f}^{2} \int \frac{d^{3} k}{(2 \pi)^{3}} f\left(\omega_{k}^{f}\right)\left[1-f\left(\omega_{k}^{f}\right)\right]=-m_{D}^{2} .
\end{aligned}
$$

Here, the contribution from the scale dependent pure vacuum self-energy given in Eq. (14) vanishes as $\lim _{q^{0}=0, \vec{q} \rightarrow 0} \operatorname{Re} \Pi_{\text {vac }}^{\mu \nu}(q)=0$ so that the Debye mass becomes independent of scale and regularization scheme. The above integral can be analytically evaluated for massless quarks $\left(m_{f}=0\right)$, and we get

$m_{D}^{2}=-\left(\frac{4 N_{c} T^{2}}{\pi^{2}}\right) \mathrm{Li}_{2}\left(-z_{q}\right) \sum_{f} e_{f}^{2}$ for massless quarks,

where $\mathrm{Li}_{2}(z)$ is the dilogarithm function. The above equation reduces to the well known expression of Debye mass

$m_{D}^{2}=N_{c} \sum_{f}\left(\frac{e_{f}^{2} T^{2}}{3}\right) \quad$ for the ideal case $\quad z_{q}=1$.

A similar result can be obtained from the semiclassical transport theory [89]

$$
\begin{aligned}
-m_{D}^{2} & =g \sum_{f} e_{f}^{2} \int \frac{d^{3} k}{(2 \pi)^{3}} \frac{\partial}{\partial \omega_{k}^{f}} f\left(\omega_{k}^{f}\right) \\
& =-g \sum_{f} e_{f}^{2} \frac{1}{T} \int \frac{d^{3} k}{(2 \pi)^{3}} f\left(\omega_{k}^{f}\right)\left[1-f\left(\omega_{k}^{f}\right)\right],
\end{aligned}
$$

where $g=2 \times 2 \times N_{c}$ is the degeneracy factor for quarkantiquark, spin, and color. It can easily be checked that the above equation boils down to Eq. (25).

Let us now turn on the external magnetic field. In this case we will take the static limit to Eq. (20), details of which are provided in Appendix B. The Debye mass in the presence of the external magnetic field comes out to be

$$
\begin{aligned}
m_{D}^{2}= & \left(\frac{N_{c} B}{\pi^{2}}\right) \sum_{f}\left|e_{f}\right|^{3} \sum_{n=0}^{\infty}\left(2-\delta_{n}^{0}\right) \\
& \times \int_{0}^{\infty} \frac{d k_{z}}{T} f\left(\omega_{k, n}^{f}\right)\left[1-f\left(\omega_{k, n}^{f}\right)\right] .
\end{aligned}
$$


Analogous results can be found from the semiclassical transport theory as well. We have the expression for Debye mass from Eq. (28) as

$$
m_{D}^{2}=g \sum_{f} e_{f}^{2} \frac{1}{T} \int \frac{d^{3} k}{(2 \pi)^{3}} f\left(\omega_{k}^{f}\right)\left[1-f\left(\omega_{k}^{f}\right)\right] .
$$

In this case, due to Landau quantization of the transverse momentum $k_{\perp}^{2}=-2 n\left|e_{f} B\right|$ of quarks/antiquarks, the dispersion relation becomes

$$
\omega_{k}^{f} \rightarrow \omega_{k, n}^{f}=\sqrt{k_{z}^{2}+m_{f}^{2}+2 n\left|e_{f} B\right|} .
$$

This in turn modifies the phase space integration in Eq. (30) as

$$
\int \frac{d^{3} k}{(2 \pi)^{3}} \rightarrow \frac{\left|e_{f} B\right|}{(2 \pi)^{3}} \sum_{n=0}^{\infty} \int_{0}^{2 \pi} d \phi \int_{-\infty}^{\infty} d k_{z}
$$

so that we get from Eq. (30)

$m_{D}^{2}=\left(\frac{B}{2 \pi^{2}}\right) \sum_{f}\left|e_{f}\right|^{3} \sum_{n=0}^{\infty} g_{n} \int_{0}^{\infty} \frac{d k_{z}}{T} f\left(\omega_{k, n}^{f}\right)\left[1-f\left(\omega_{k, n}^{f}\right)\right]$,

where the degeneracy factor $g_{n}$ is dependent on the Landau level index $n$. It is well known [90] that the lowest Landau level (LLL) is spin nondegenerate, which implies that $g_{n}=2 \times\left(2-\delta_{n}^{0}\right) \times N_{c}$. Substituting $g_{n}$ in the above expression, we recover Eq. (29).

\section{ELECTROMAGNETIC SPECTRAL FUNCTION}

The electromagnetic spectral function $\rho^{q \bar{q}}(q)$ due to the quark loop is defined as

$$
\rho^{q \bar{q}}(q)=\frac{1}{4 \pi \alpha} g_{\mu \nu} \operatorname{Im} \bar{\Pi}^{\mu \nu}(q) .
$$

Let us first calculate $\rho^{q \bar{q}}(q)$ for the zero magnetic field case that is obtained from Eq. (12) after contracting with $g_{\mu \nu}$. The four terms in Eq. (12) with the four Dirac delta functions are termed as Unitary-I, Unitary-II, Landau-II, and Landau-I cuts, respectively, according to their appearance in that equation. These terms are nonvanishing in certain kinematic domains as can be read from Appendix D. The Unitary-I term is nonvanishing for $\sqrt{\vec{q}^{2}+4 m_{f}^{2}} \leq q^{0}<\infty$, whereas the Unitary-II term is nonvanishing for $-\infty<q^{0} \leq$ $-\sqrt{\vec{q}^{2}+4 m_{f}^{2}}$. Both the Landau terms have their corresponding kinematic region as $\left|q^{0}\right| \leq|\vec{q}|$. These different cuts correspond to different physical processes such as decay and scattering. For example, the Unitary-I cut corresponds to the decay of a photon with energy $q^{0}>\sqrt{\vec{q}^{2}+4 m_{f}^{2}}$ into a quark-antiquark pair (which is the threshold energy for a pair creation) and also the time reversed process that a quarkantiquark annihilates to make a photon. Similarly the Landau cuts correspond to the absorption of a photon with energy $\left|q^{0}\right| \leq|\vec{q}|$ due to scattering with a quark producing another quark in the final state and also the time reversed process that a quark emits a photon in the medium. If we restrict ourselves to the physical time such as the region defined in terms of $q^{0}>0$ and $q^{2}>0$, then only the Unitary-I cut contributes. Therefore, in the physical region, processes such as photon decay and formation occur via the Unitary-I cut, whereas the scattering and emission processes do not occur.

We then evaluate the $d(\cos \theta)$ integrals in Eq. (12) by using the Dirac delta functions [56] in order to simplify the expression of the spectral function and impose the kinematic restrictions discussed above to get

$$
\begin{aligned}
\rho^{q \bar{q}}\left(q^{0}, \vec{q}\right)= & \frac{1}{4 \pi \alpha} \operatorname{sign}\left(q^{0}\right) \tanh \left(\frac{\beta q^{0}}{2}\right) \sum_{f} e_{f}^{2} N_{c} \frac{1}{16 \pi|\vec{q}|}\left[\int_{\omega_{-}^{f}}^{\omega_{+}^{f}} d \omega_{k}^{f} U_{1}^{f}\left(\cos \theta=\cos \theta_{0}\right) \Theta\left(q^{0}-\sqrt{\vec{q}^{2}+4 m_{f}^{2}}\right)\right. \\
& +\int_{-\omega_{+}^{f}}^{-\omega_{-}^{f}} d \omega_{k}^{f} U_{2}^{f}\left(\cos \theta=\cos \theta_{0}^{\prime}\right) \Theta\left(-q^{0}-\sqrt{\vec{q}^{2}+4 m_{f}^{2}}\right)+\int_{-\omega_{+}^{f}}^{\infty} d \omega_{k}^{f} L_{1}^{f}\left(\cos \theta=\cos \theta_{0}^{\prime}\right) \Theta\left(-\left|q^{0}\right|+|\vec{q}|\right) \\
& \left.+\int_{\omega_{-}^{f}}^{\infty} d \omega_{k}^{f} L_{2}^{f}\left(\cos \theta=\cos \theta_{0}\right) \Theta\left(-\left|q^{0}\right|+|\vec{q}|\right)\right],
\end{aligned}
$$

where

$$
\begin{gathered}
\omega_{ \pm}^{f}=\frac{1}{2 q^{2}}\left[q^{0} q^{2} \pm|\vec{q}| \lambda^{1 / 2}\left(q^{2}, m_{f}^{2}, m_{f}^{2}\right)\right], \\
U_{1}=\left\{1-f\left(\omega_{k}^{f}\right)-f\left(\omega_{p}^{f}\right)+2 f\left(\omega_{k}^{f}\right) f\left(\omega_{p}^{f}\right)\right\} g_{\mu \nu} N_{f}^{\mu \nu}\left(k^{0}=-\omega_{k}^{f}\right), \\
U_{2}=\left\{1-f\left(\omega_{k}^{f}\right)-f\left(\omega_{p}^{f}\right)+2 f\left(\omega_{k}^{f}\right) f\left(\omega_{p}^{f}\right)\right\} g_{\mu \nu} N_{f}^{\mu \nu}\left(k^{0}=\omega_{k}^{f}\right),
\end{gathered}
$$




$$
\begin{gathered}
L_{1}=\left\{-f\left(\omega_{k}^{f}\right)-f\left(\omega_{p}^{f}\right)+2 f\left(\omega_{k}^{f}\right) f\left(\omega_{p}^{f}\right)\right\} g_{\mu \nu} N_{f}^{\mu \nu}\left(k^{0}=\omega_{k}^{f}\right), \\
L_{2}=\left\{-f\left(\omega_{k}^{f}\right)-f\left(\omega_{p}^{f}\right)+2 f\left(\omega_{k}^{f}\right) f\left(\omega_{p}^{f}\right)\right\} g_{\mu \nu} N_{f}^{\mu \nu}\left(k^{0}=-\omega_{k}^{f}\right), \\
\cos \theta_{0}=\left(\frac{-2 q^{0} \omega_{k}^{f}+q^{2}}{2|\vec{q}||\vec{k}|}\right), \\
\cos \theta_{0}^{\prime}=\left(\frac{2 q^{0} \omega_{k}^{f}+q^{2}}{2|\vec{q}||\vec{k}|}\right)
\end{gathered}
$$

with $\lambda(x, y, z)=x^{2}+y^{2}+z^{2}-2 x y-2 y z-2 z x$ being the Källén function. It is easy to check from Eq. (13) that $g_{\mu \nu} N_{f}^{\mu \nu}=8\left(2 m_{f}^{2}-k^{2}-k \cdot q\right)$.

Let us now turn on the external magnetic field. In this case, the calculation spectral function is simplified if we consider the transverse momentum $q_{\perp}$ of the photon to be zero. This has been provided in Appendix C, and we get from Eq. (C7)

$$
\begin{aligned}
\rho^{q \bar{q}}\left(q^{0}, q_{z}\right)= & \frac{1}{4 \pi \alpha} \operatorname{sign}\left(q^{0}\right) \tanh \left(\frac{\beta q^{0}}{2}\right) \sum_{f} e_{f}^{2} N_{c} \sum_{n=0}^{\infty} \sum_{l=(n-1)}^{(n+1)} \pi \int_{-\infty}^{\infty} \frac{d k_{z}}{2 \pi} \frac{1}{4 \omega_{k, l}^{f} \omega_{p, n}^{f}}\left[\tilde{U}_{1, n l}^{f} \delta\left(q^{0}-\omega_{k, l}^{f}-\omega_{p, n}^{f}\right)\right. \\
& \left.+\tilde{U}_{2, n l}^{f} \delta\left(q^{0}+\omega_{k, l}^{f}+\omega_{p, n}^{f}\right)+\tilde{L}_{1, n l}^{f} \delta\left(q^{0}+\omega_{k, l}^{f}-\omega_{p, n}^{f}\right)+\tilde{L}_{2, n l}^{f} \delta\left(q^{0}-\omega_{k, l}^{f}+\omega_{p, n}^{f}\right)\right] .
\end{aligned}
$$

Let us now discuss the analytic structure of the spectral function in the presence of the external magnetic field. As can be obtained from Appendix D, in this case, the kinematic regions for Unitary-I and Unitary-II cuts are, respectively, $\sqrt{q_{z}^{2}+4 m_{f}^{2}} \leq q^{0}<\infty$ and $-\infty<q^{0} \leq-\sqrt{q_{z}^{2}+4 m_{f}^{2}}$, whereas the corresponding region for both the Landau cuts is $\left|q^{0}\right| \leq \sqrt{q_{z}^{2}+\left(m_{f}-\sqrt{m_{f}^{2}+2\left|e_{f} B\right|}\right)^{2}}$. Hence, if we restrict ourselves to the physical timelike regions in terms of $q^{0}>0$ and $q^{2}>0$, then along with the UnitaryI cut, both the Landau cuts contribute. The appearance of these Landau cuts is a purely magnetic field effect as can be noticed that, at $B \rightarrow 0$, the Landau cuts disappear from the physical timelike region. Physically this means that, in addition to the decay/formation processes, scattering/emission can also happen in the presence of the external magnetic field. A photon can scatter with a quark from the lower Landau level to get absorbed producing another quark on a higher Landau level, and the time reversed process such as a quark from a higher Landau level can emit a photon and goes down to a lower Landau level. As mentioned earlier, this type of process cannot happen in the absence of the external magnetic field. We will see in the next section that the appearance of the Landau cuts will enhance the low invariant mass dilepton production rate.

Let us now simplify Eq. (43) by evaluating the $d k_{z}$ integral using the Dirac delta functions and impose the kinematic restrictions as discussed above. The result is

$$
\begin{aligned}
\rho^{q \bar{q}}\left(q^{0}, q_{z}\right)= & \frac{1}{4 \pi \alpha} \operatorname{sign}\left(q^{0}\right) \tanh \left(\frac{\beta q^{0}}{2}\right) \sum_{f} e_{f}^{2} N_{c} \sum_{n=0}^{\infty} \sum_{l=(n-1)}^{(n+1)} \frac{1}{4 \lambda^{1 / 2}\left(q_{\|}^{2}, m_{f, l}^{2}, m_{f, n}^{2}\right)} \\
& \times \sum_{\tilde{k}_{z} \in \tilde{k}_{z}^{ \pm}}\left[\tilde{U}_{1, n l}^{f}\left(k_{z}=\tilde{k}_{z}\right) \Theta\left(q^{0}-\sqrt{q_{z}^{2}+\left(m_{f, l}+m_{f, n}\right)^{2}}\right)+\tilde{U}_{2, n l}^{f}\left(k_{z}=\tilde{k}_{z}\right) \Theta\left(-q^{0}-\sqrt{q_{z}^{2}+\left(m_{f, l}+m_{f, n}\right)^{2}}\right)\right. \\
& +\tilde{L}_{1, n l}^{f}\left(k_{z}=\tilde{k}_{z}\right) \Theta\left(q^{0}-\min \left(q_{z}, E_{ \pm}^{\prime}\right)\right) \Theta\left(-q^{0}+\max \left(q_{z}, E_{ \pm}^{\prime}\right)\right) \\
& \left.+\tilde{L}_{2, n l}^{f}\left(k_{z}=\tilde{k}_{z}\right) \Theta\left(-q^{0}-\min \left(q_{z}, E_{ \pm}^{\prime}\right)\right) \Theta\left(q^{0}+\max \left(q_{z}, E_{ \pm}^{\prime}\right)\right)\right]
\end{aligned}
$$

where $\quad \tilde{k}_{z}^{ \pm}=\frac{1}{2 q_{\|}^{2}}\left[-y q_{z} \pm\left|q^{0}\right| \lambda^{1 / 2}\left(q_{\|}^{2}, m_{f, l}^{2}, m_{f, n}^{2}\right)\right], \quad y=$ $\left(q_{\|}^{2}+m_{f, l}^{2}-m_{f, n}^{2}\right), \quad \tilde{\omega}_{k, l}^{f}=\sqrt{\tilde{k}_{z}^{2}+m_{f, l}^{2}}, \quad E_{ \pm}^{\prime}=\frac{m_{f, l}-m_{f, n}}{\left|m_{f, l} \pm m_{f, n}\right|} \times$ $\sqrt{q_{z}^{2}+\left(m_{f, l} \pm m_{f, n}\right)^{2}}$, and $m_{f, l}$ is defined in Eq. (22).

\section{DILEPTON PRODUCTION RATE AT ZERO EXTERNAL MAGNETIC FIELD}

In order to calculate the DPR from a hot QCD medium, we follow the standard procedure given in Ref. [56] and 
consider an initial state $|i\rangle=\left|I\left(p_{I}\right)\right\rangle$ of quark/antiquark with momentum $p_{I}$, which goes to a final state $|f\rangle=$ $\left|F\left(p_{F}\right) l^{+}\left(p_{+}, s_{+}\right) l^{-}\left(p_{-}, s_{-}\right)\right\rangle$containing the quark/antiquark with momentum $p_{F}$ plus dilepton with momenta $p_{+}, p_{-}$ and spin $s_{+}, s_{-}$. The probability amplitude for transition $|i\rangle \rightarrow|f\rangle$ is $\langle f|\hat{S}| i\rangle$ where $\hat{S}$ is the scattering matrix operator

$$
\hat{S}=\mathcal{T}\left[\exp \left\{i \int \mathcal{L}_{\text {int }}(x) d^{4} x\right\}\right]
$$

with

$$
\mathcal{L}_{\text {int }}(x)=\left[j^{\mu}(x)+J^{\mu}(x)\right] A_{\mu}(x) .
$$

Here $\mathcal{T}$ is the time ordering symbol, $j^{\mu}(x)$ is the lepton current, $J^{\mu}(x)$ is the quark/antiquark current, and $A_{\mu}(x)$ is the photon field. The lepton and quark/antiquark currents are given by

$$
\begin{gathered}
j^{\mu}(x)=-e \bar{\psi}(x) \gamma^{\mu} \psi(x), \\
J^{\mu}(x)=\sum_{f} e_{f} \bar{q}_{f}(x) \gamma^{\mu} q_{f}(x),
\end{gathered}
$$

where $\psi(x)$ and $q_{f}(x)$ are, respectively, the lepton and quark fields. Expanding $\hat{S}$ up to second order and after some simplifications, the nontrivial contribution to the $S$-matrix element comes from

$$
\begin{aligned}
\langle f|\hat{S}| i\rangle= & -\iint d^{4} x_{1} d^{4} x_{2}\left\langle F\left|J_{\mu}\left(x_{1}\right)\right| I\right\rangle \\
& \times\left\langle l^{+} l^{-}\left|j_{\nu}\left(x_{2}\right)\right| 0\right\rangle \Delta_{F}^{\mu \nu}\left(x_{1}-x_{2}\right),
\end{aligned}
$$

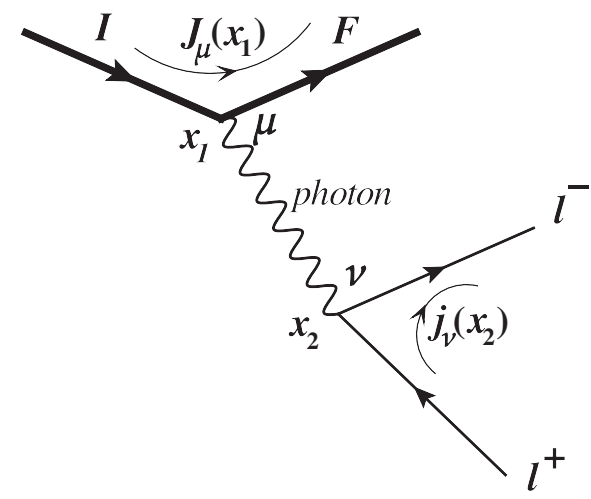

FIG. 2. Diagrammatic representation of the dilepton production amplitude from Eq. (49).

where $\Delta_{F}^{\mu \nu}\left(x_{1}-x_{2}\right)=\left\langle 0\left|\mathcal{T} A^{\mu}\left(x_{1}\right) A^{\nu}\left(x_{2}\right)\right| 0\right\rangle$ is the coordinate space vacuum Feynman photon propagator which can be Fourier transformed as

$$
\Delta_{F}^{\mu \nu}\left(x_{1}-x_{2}\right)=\int \frac{d^{4} q}{(2 \pi)^{4}} e^{-i q \cdot\left(x_{1}-x_{2}\right)}\left(-i \Delta_{F}^{\mu \nu}(q)\right)
$$

in which

$$
\Delta_{F}^{\mu \nu}(q)=-g^{\mu \nu}\left(\frac{-1}{q^{2}+i \epsilon}\right)
$$

is the momentum space vacuum Feynman photon propagator. Figure 2 shows the diagrammatic representation of the dilepton production amplitude as given in Eq. (49). In Eq. (49), a translation of the leptonic current operator $j_{\nu}\left(x_{2}\right)$ to the origin leads to the following simplification of its matrix element:

$$
\left\langle l^{+} l^{-}\left|j_{\nu}\left(x_{2}\right)\right| 0\right\rangle=\left\langle l^{+} l^{-}\left|e^{i \hat{P} \cdot x_{2}} j_{\nu}(0) e^{-i \hat{P} \cdot x_{2}}\right| 0\right\rangle=e^{i\left(p_{+}+p_{-}\right) \cdot x_{2}}\left\langle l^{+} l^{-}\left|j_{\nu}(0)\right| 0\right\rangle .
$$

We now substitute Eqs. (50) and (52) into Eq. (49) and perform the $d^{4} q$ integral, which gives a Dirac delta function $\delta^{4}\left(q+p_{+}+p_{-}\right)$. This delta function is in turn used to perform the $d^{4} x_{2}$ integral, and we are left with

$$
\langle f|\hat{S}| i\rangle=i \int d^{4} x_{1} e^{i\left(p_{+}+p_{-}\right) \cdot x_{1}}\left\langle F\left|J_{\mu}\left(x_{1}\right)\right| I\right\rangle\left\langle l^{+} l^{-}\left|j_{\nu}(0)\right| 0\right\rangle \Delta_{F}^{\mu \nu}\left(-p_{+}-p_{-}\right) .
$$

Taking the absolute square of the above equation, we get

$$
\begin{aligned}
|\langle f|\hat{S}| i\rangle|^{2}= & \iint d^{4} x_{1} d^{4} x e^{i\left(p_{+}+p_{-}\right) \cdot\left(x_{1}-x\right)}\left\langle F\left|J_{\mu}\left(x_{1}\right)\right| I\right\rangle\left\langle I\left|J_{\alpha}^{\dagger}(x)\right| F\right\rangle \\
& \times\left\langle l^{+} l^{-}\left|j_{\nu}(0)\right| 0\right\rangle\left\langle 0\left|j_{\beta}^{\dagger}(0)\right| l^{+} l^{-}\right\rangle \Delta_{F}^{\mu \nu}\left(-p_{+}-p_{-}\right) \Delta_{F}^{\alpha \beta}\left(-p_{+}-p_{-}\right) .
\end{aligned}
$$

Translating the quark/antiquark current operator $J_{\alpha}^{\dagger}(x)$ to the origin, we can write its matrix element as

$$
\left\langle I\left|J_{\alpha}^{\dagger}(x)\right| F\right\rangle=e^{i\left(p_{I}-p_{F}\right) \cdot x}\left\langle I\left|J_{\alpha}^{\dagger}(0)\right| F\right\rangle .
$$

We now substitute the above matrix element as well as the momentum space photon propagator from Eq. (51) into Eq. (54) and impose the momentum conservation $p_{I}=p_{F}+p_{+}+p_{-}$to get 


$$
|\langle f|\hat{S}| i\rangle|^{2}=\iint d^{4} x_{1} d^{4} x \frac{e^{i\left(p_{+}+p_{-}\right) \cdot x_{1}}}{\left(p_{+}+p_{-}\right)^{4}}\left\langle F\left|J_{\mu}\left(x_{1}\right)\right| I\right\rangle\left\langle I\left|J_{\nu}^{\dagger}(0)\right| F\right\rangle\left\langle l^{+} l^{-}\left|j^{\mu}(0)\right| 0\right\rangle\left\langle 0\left|j^{\dagger \nu}(0)\right| l^{+} l^{-}\right\rangle
$$

The dilepton multiplicity $(N)$ from the thermal QGP medium is given by [56]

$$
N=\sum_{s_{+}, s_{-}} \int \frac{d^{3} p_{+}}{(2 \pi)^{3} 2 E_{+}} \int \frac{d^{3} p_{-}}{(2 \pi)^{3} 2 E_{-}} \frac{1}{\mathcal{Z}} \sum_{I, F} e^{-\beta E_{I}}|\langle f|\hat{S}| i\rangle|^{2},
$$

where $E_{ \pm}=\sqrt{m^{2}+\vec{p}_{ \pm}^{2}}, m$ is the lepton mass, and $\mathcal{Z}$ is the partition function of the system. We now substitute Eq. (56) into the above equation and get

$$
\begin{aligned}
N= & \sum_{s_{+}, s_{-}} \int \frac{d^{3} p_{+}}{(2 \pi)^{3} 2 E_{+}} \int \frac{d^{3} p_{-}}{(2 \pi)^{3} 2 E_{-}}\left\langle l^{+} l^{-}\left|j^{\mu}(0)\right| 0\right\rangle\left\langle 0\left|j^{\dagger \nu}(0)\right| l^{+} l^{-}\right\rangle \iint d^{4} x d^{4} x_{1} \frac{e^{i\left(p_{+}+p_{-}\right) \cdot x_{1}}}{\left(p_{+}+p_{-}\right)^{4}} \\
& \times\left[\frac{1}{\mathcal{Z}} \sum_{I, F} e^{-\beta E_{I}}\left\langle F\left|J_{\mu}\left(x_{1}\right)\right| I\right\rangle\left\langle I\left|J_{\nu}^{\dagger}(0)\right| F\right\rangle\right] .
\end{aligned}
$$

The quantity within the square bracket in the above equation can be simplified using the momentum conservation $p_{I}=p_{F}+p_{+}+p_{-}$and the completeness relation of the initial states $\sum_{I}|I\rangle\langle I|=\mathbb{1}$ so that

$$
\begin{gathered}
\frac{1}{\mathcal{Z}} \sum_{I, F} e^{-\beta E_{I}}\left\langle F\left|J_{\mu}\left(x_{1}\right)\right| I\right\rangle\left\langle I\left|J_{\nu}^{\dagger}(0)\right| F\right\rangle \\
=e^{-\beta\left(E_{+}+E_{-}\right)}\left\langle J_{\mu}\left(x_{1}\right) J_{\nu}^{\dagger}(0)\right\rangle,
\end{gathered}
$$

where \langle\rangle denotes the ensemble average. Substituting the above equation into Eq. (58) and inserting the identity $1=$ $\int d^{4} q \delta^{4}\left(q-p_{+}-p_{-}\right)$in the RHS of Eq. (58) we get

$$
N=\iint d^{4} x d^{4} q \frac{e^{-\beta q^{0}}}{(2 \pi)^{4} q^{4}} W_{\mu \nu}^{+}(q) L^{+\mu \nu}(q)
$$

where

$$
\begin{gathered}
W_{\mu \nu}^{+}(q)=\int d^{4} x e^{i q \cdot x}\left\langle J_{\mu}(x) J_{\nu}^{\dagger}(0)\right\rangle, \\
L_{\mu \nu}^{+}(q)=(2 \pi)^{4} \sum_{s_{+}, s_{-}} \int \frac{d^{3} p_{+}}{(2 \pi)^{3} 2 E_{+}} \int \frac{d^{3} p_{-}}{(2 \pi)^{3} 2 E_{-}} \\
\times \delta^{4}\left(q-p_{+}-p_{-}\right)\left\langle l^{+} l^{-}\left|j_{\mu}(0)\right| 0\right\rangle\left\langle 0\left|j_{\nu}^{\dagger}(0)\right| l^{+} l^{-}\right\rangle .
\end{gathered}
$$

Thus, the DPR, i.e., the dilepton production per unit phase space volume $d^{4} x d^{4} q$, is given by [from Eq. (60)]

$$
\mathrm{DPR}=\left(\frac{d N}{d^{4} q d^{4} x}\right)=\frac{e^{-\beta q^{0}}}{(2 \pi)^{4} q^{4}} W_{\mu \nu}^{+}(q) L^{+\mu \nu}(q) .
$$

In order to calculate $W_{\mu \nu}^{+}(q)$, we use the RTF of TFT in which it is related to the imaginary part of the 11 component of the Fourier transform of time order current-current correlator [56,82],

$$
W_{\mu \nu}^{+}(q)=\left(\frac{1}{e^{-\beta q^{0}}+1}\right) 2 \operatorname{Im} W_{\mu \nu}^{11}(q),
$$

where

$$
W_{\mu \nu}^{11}(q)=i \int d^{4} x e^{i q \cdot x}\left\langle\mathcal{T} J_{\mu}(x) J_{\nu}^{\dagger}(0)\right\rangle .
$$

The calculation of $W_{\mu \nu}^{11}(q)$ is provided in Appendix E, and the final result can be read from Eq. (E6), which comes out to be exactly $(-1)$ times the 11 component of the real time thermal photon self-energy given in Eq. (6). Thus the DPR becomes

$$
\begin{aligned}
\mathrm{DPR} & =\left(\frac{d N}{d^{4} q d^{4} x}\right) \\
& =\frac{e^{-\beta q^{0}}}{(2 \pi)^{4} q^{4}}\left(\frac{-2}{e^{-\beta q^{0}}+1}\right) \operatorname{Im} \Pi_{\nu \mu}^{11}(q) L^{+\mu \nu}(q) .
\end{aligned}
$$

The lepton tensor $L_{\mu \nu}^{+}(q)$ in Eq. (62) can be expressed in a more convenient form that is analogous to $W_{\mu \nu}^{+}(q)$ in Eq. (61). For that, we write

$$
\begin{aligned}
& (2 \pi)^{4} \delta^{4}\left(q-p_{+}-p_{-}\right)\left\langle 0\left|j_{\nu}^{\dagger}(0)\right| l^{+} l^{-}\right\rangle \\
& \quad=\int d^{4} x e^{i x \cdot\left(q-p_{+}-p_{-}\right)}\left\langle 0\left|j_{\nu}^{\dagger}(0)\right| l^{+} l^{-}\right\rangle \\
& \quad=\int d^{4} x e^{i q \cdot x}\left\langle 0\left|j_{\nu}^{\dagger}(x)\right| l^{+} l^{-}\right\rangle,
\end{aligned}
$$

where the lepton current operator $j_{\nu}^{\dagger}(0)$ has been translated from the origin to $x$. Substituting the above equation into Eq. (62) we get 


$$
\begin{aligned}
L_{\mu \nu}^{+}(q)= & \int d^{4} x e^{i q \cdot x} \sum_{s_{+}, s_{-}} \int \frac{d^{3} p_{+}}{(2 \pi)^{3} 2 E_{+}} \\
& \times \int \frac{d^{3} p_{-}}{(2 \pi)^{3} 2 E_{-}}\left\langle 0\left|j_{\nu}^{\dagger}(x)\right| l^{+} l^{-}\right\rangle\left\langle l^{+} l^{-}\left|j_{\mu}(0)\right| 0\right\rangle .
\end{aligned}
$$

We now use the completeness relation for the two-particle leptonic state

$$
\mathbb{1}=\sum_{s_{+}, s_{-}} \int \frac{d^{3} p_{+}}{(2 \pi)^{3} 2 E_{+}} \int \frac{d^{3} p_{-}}{(2 \pi)^{3} 2 E_{-}}\left|l^{+} l^{-}\right\rangle\left\langle l^{+} l^{-}\right|
$$

so that Eq. (68) becomes

$$
L_{\mu \nu}^{+}(q)=\int d^{4} x e^{i q \cdot x}\left\langle 0\left|j_{\nu}^{\dagger}(x) j_{\mu}(0)\right| 0\right\rangle,
$$

which is analogous to the expression of $W_{\mu \nu}^{+}$in Eq. (61) where we have an ensemble average instead of the vacuum expectation value. Thus, similar to Eq. (64), $L_{\mu \nu}^{+}(q)$ in the above equation can be related to the imaginary part of the Fourier transform of the time order leptonic current-current correlator $[56,82]$ as

$$
L_{\mu \nu}^{+}(q)=2 \operatorname{Im} L_{\mu \nu}(q)
$$

where

$$
L_{\mu \nu}(q)=i \int d^{4} x e^{i q \cdot x}\left\langle 0\left|\mathcal{T} j_{\nu}^{\dagger}(x) j_{\mu}(0)\right| 0\right\rangle .
$$

The calculation of $L_{\mu \nu}$ is provided in Appendix F, and it comes out to be exactly $(-1)$ times the photon vacuum selfenergy $\pi_{\mu \nu}(q)$ due to the $l^{+} l^{-}$loop given by

$-L_{\mu \nu}(q)=\pi^{\mu \nu}(q)=-i e^{2} \int \frac{d^{4} k}{(2 \pi)^{4}} \operatorname{Tr}\left[\gamma^{\nu} S_{l}(q+k) \gamma^{\mu} S_{l}(k)\right]$,

where $S_{l}(p)=\frac{-(\not+m)}{p^{2}-m^{2}+i \varepsilon}$ is the vacuum lepton Feynman propagator. It is worth mentioning that the forms of $L_{\mu \nu}^{+}(q)$ in both Eqs. (68) and (71) boil down to the same analytical expression that is shown in Appendix G. The calculation of $L_{\mu \nu}^{+}(q)$ from Eq. (68) requires the knowledge of spin sums over leptonic spinors, whereas its calculation from (71) does not require the same. We will see that, the particular form of $L_{\mu \nu}^{+}(q)$ in Eq. (71) together with Eq. (73) will be of more convenience while introducing the external magnetic field. For that case, the charged lepton propagators $S_{l}(p)$ in the above equation will be replaced by the Schwinger proper-time one, and thus a knowledge of spin sum in the presence of the external magnetic field will not be required.

We now substitute $L_{\mu \nu}^{+}(q)$ from Eqs. (71) and (73) into Eq. (66) followed by using Eq. (9) to obtain
$\mathrm{DPR}=\left(\frac{d N}{d^{4} q d^{4} x}\right)=\frac{4}{(2 \pi)^{4} q^{4}}\left(\frac{1}{e^{\beta q^{0}}-1}\right) \operatorname{Im} \bar{\Pi}_{\nu \mu}(q) \operatorname{Im} \pi^{\mu \nu}(q)$.

The conservation of the lepton current requires that $q_{\mu} \pi^{\mu \nu}(q)=0$, which fixes the Lorentz structure of $\pi^{\mu \nu}$ as

$$
\pi^{\mu \nu}(q)=\left(g^{\mu \nu}-\frac{q^{\mu} q^{\nu}}{q^{2}}\right) \frac{1}{3} \pi_{\alpha}^{\alpha}(q) .
$$

Substituting the above equation into Eq. (74), and imposing the conservation of the quark/antiquark current by means of $q^{\mu} \bar{\Pi}_{\mu \nu}(q)=0$, we get

$$
\begin{aligned}
\mathrm{DPR} & =\left(\frac{d N}{d^{4} q d^{4} x}\right) \\
& =\frac{4}{3(2 \pi)^{4} q^{4}}\left(\frac{1}{e^{\beta q^{0}}-1}\right) \operatorname{Im} \bar{\Pi}_{\mu}^{\mu}(q) \operatorname{Im} \pi_{\nu}^{\nu}(q) .
\end{aligned}
$$

Let us now finally express the DPR in terms of electromagnetic spectral functions $\rho^{q \bar{q}}$ and $\rho^{\bar{l}}$ as

$\mathrm{DPR}=\left(\frac{d N}{d^{4} q d^{4} x}\right)=\frac{4 \alpha^{2}}{3 \pi^{2} q^{4}}\left(\frac{1}{e^{\beta q^{0}}-1}\right) \rho^{q \bar{q}}(q) \rho^{\bar{l}}(q)$,

where $\rho^{q \bar{q}}$ is defined in Eq. (34) and $\rho^{l \bar{l}}$ is defined as

$$
\rho^{l \bar{l}}(q)=\frac{1}{4 \pi \alpha} \operatorname{Im} \pi_{\mu}^{\mu}(q) .
$$

Since we have already calculated $\rho^{q \bar{q}}$ in Eq. (35), it is trivial to write down the expression for $\rho^{l \bar{l}}$ by replacing $N_{c} \rightarrow 1$, $T \rightarrow 0, \sum_{f} e_{f}^{2} \rightarrow e^{2}$, and $m_{f} \rightarrow m$ in Eq. (35). Considering the dileptons with physical momenta $\left(q^{2}>0, q^{0}>0\right)$, only the Unitary-I cut contributes and we get

$$
\begin{aligned}
\rho^{l \bar{l}}(q)= & \frac{1}{16 \pi|\vec{q}|} \int_{\omega_{-}}^{\omega_{+}} d \omega_{k} U_{1}\left(\cos \theta=\cos \theta_{0}\right) \\
& \times \Theta\left(q^{0}-\sqrt{\vec{q}^{2}+4 m^{2}}\right)
\end{aligned}
$$

where

$$
\begin{gathered}
\omega_{ \pm}=\frac{1}{2 q^{2}}\left[q^{0} q^{2} \pm|\vec{q}| \lambda^{1 / 2}\left(q^{2}, m^{2}, m^{2}\right)\right] \\
U_{1}=\left.8\left(2 m^{2}-k^{2}-k \cdot q\right)\right|_{k^{0}=-\omega_{k}} \\
\cos \theta_{0}=\left(\frac{-2 q^{0} \omega_{k}+q^{2}}{2|\vec{q}||\vec{k}|}\right)
\end{gathered}
$$

with $\omega_{k}=\sqrt{\vec{k}^{2}+m^{2}}$. Substituting Eqs. (80)-(82) into Eq. (79) and performing the $d \omega_{k}$ integral, we arrive at 
$\rho^{\bar{l}}(q)=\frac{q^{2}}{4 \pi}\left(1+\frac{2 m^{2}}{q^{2}}\right)\left(1-\frac{4 m^{2}}{q^{2}}\right)^{1 / 2} \Theta\left(q^{2}-4 m^{2}\right)$.

Substituting $\rho^{\bar{l}}(q)$ from the above equation into Eq. (77), we obtain the final expression of DPR from QGP under the zero external magnetic field as

$$
\begin{aligned}
\mathrm{DPR}= & \left(\frac{d N}{d^{4} q d^{4} x}\right) \\
= & \frac{\alpha^{2}}{3 \pi^{3} q^{2}}\left(1+\frac{2 m^{2}}{q^{2}}\right)\left(1-\frac{4 m^{2}}{q^{2}}\right)^{1 / 2} \\
& \times\left(\frac{1}{e^{\beta q^{0}}-1}\right) \rho^{q \bar{q}}(q) \Theta\left(q^{2}-4 m^{2}\right) .
\end{aligned}
$$

The presence of the unit step function in the above equation restricts the kinematic region where the DPR is nonzero.

\section{DILEPTON PRODUCTION RATE UNDER EXTERNAL MAGNETIC FIELD}

In order to calculate the DPR from a magnetized QGP medium, we start with Eq. (74),

$$
\begin{aligned}
\mathrm{DPR} & =\left(\frac{d N}{d^{4} q d^{4} x}\right) \\
& =\frac{4}{(2 \pi)^{4} q^{4}}\left(\frac{1}{e^{\beta q^{0}}-1}\right) \operatorname{Im} \bar{\Pi}_{\nu \mu}(q) \operatorname{Im} \pi^{\mu \nu}(q),
\end{aligned}
$$

where the effect of the external magnetic field will be entered through the photon polarization tensors $\bar{\Pi}_{\mu \nu}(q)$ and $\bar{\pi}_{\mu \nu}(q)$ calculated in the presence of the magnetic field. For the sake of simplicity in analytic calculation, we take the transverse momenta of the photon to be zero, i.e., $q_{\perp}=0$, so that, in the presence of the external magnetic field, the Lorentz structure of $\pi^{\mu \nu}\left(q_{\|}\right)$will be

$\pi^{\mu \nu}\left(q_{\|}\right)=\left(g_{\|}^{\mu \nu}-\frac{q_{\|}^{\mu} q_{\|}^{\nu}}{q_{\|}^{2}}\right)\left(\pi_{\alpha \beta} g_{\|}^{\alpha \beta}\right)+g_{\perp}^{\mu \nu}\left(\frac{1}{2} \pi_{\alpha \beta} g_{\perp}^{\alpha \beta}\right)$.

Substituting the above equation into Eq. (85) and imposing the conservation of quark/antiquark current $\left(q_{\|}^{\mu} \bar{\Pi}_{\mu \nu}\left(q_{\|}\right)=0\right)$, we get

$$
\mathrm{DPR}=\left(\frac{d N}{d^{4} q d^{4} x}\right)=\frac{4}{(2 \pi)^{4} q_{\|}^{4}}\left(\frac{1}{e^{\beta q^{0}}-1}\right)\left[g_{\|}^{\mu \nu} g_{\|}^{\alpha \beta} \operatorname{Im} \bar{\Pi}_{\nu \mu}\left(q_{\|}\right) \operatorname{Im} \pi_{\alpha \beta}\left(q_{\|}\right)+\frac{1}{2} g_{\perp}^{\mu \nu} g_{\perp}^{\alpha \beta} \operatorname{Im} \bar{\Pi}_{\nu \mu}\left(q_{\|}\right) \operatorname{Im} \pi_{\alpha \beta}\left(q_{\|}\right)\right] .
$$

We define the longitudinal and transverse spectral functions as

$$
\begin{array}{rlrl}
\rho_{\|}^{q \bar{q}}\left(q_{\|}\right) & =\frac{1}{4 \pi \alpha} g_{\|}^{\mu \nu} \operatorname{Im} \bar{\Pi}_{\nu \mu}\left(q_{\|}\right), & & \rho_{\perp}^{q \bar{q}}\left(q_{\|}\right)=\frac{1}{4 \pi \alpha} g_{\perp}^{\mu \nu} \operatorname{Im} \bar{\Pi}_{\nu \mu}\left(q_{\|}\right), \\
\rho_{\|}^{l \bar{l}}\left(q_{\|}\right)=\frac{1}{4 \pi \alpha} g_{\|}^{\mu \nu} \operatorname{Im} \pi_{\mu \nu}\left(q_{\|}\right), & \rho_{\perp}^{l \bar{l}}\left(q_{\|}\right) & =\frac{1}{4 \pi \alpha} g_{\perp}^{\mu \nu} \operatorname{Im} \pi_{\mu \nu}\left(q_{\|}\right)
\end{array}
$$

in terms of which the DPR may be expressed as

$$
\mathrm{DPR}=\left(\frac{d N}{d^{4} q d^{4} x}\right)=\frac{4 \alpha^{2}}{\pi^{2} q_{\|}^{4}}\left(\frac{1}{e^{\beta q^{0}}-1}\right)\left[\rho_{\|}^{q \bar{q}}\left(q_{\|}\right) \rho_{\|}^{\bar{l} \bar{l}}\left(q_{\|}\right)+\frac{1}{2} \rho_{\perp}^{q \bar{q}}\left(q_{\|}\right) \rho_{\perp}^{\bar{l} \bar{l}}\left(q_{\|}\right)\right]
$$

The next step is to calculate the longitudinal and transverse spectral functions defined in Eqs. (88) and (89). We have already calculated $\rho^{q \bar{q}}\left(q_{\|}\right)=\rho_{\|}^{q \bar{q}}\left(q_{\|}\right)+\rho_{\perp}^{q \bar{q}}\left(q_{\|}\right)$in Eq. (44), which can be split into the transverse and longitudinal parts. This is done by splitting Eq. (C12) into a longitudinal and a transverse part as

$$
\begin{aligned}
N_{f, n l}\left(q_{\|}, k_{\|}\right) & =(-1)^{n+l}\left(\frac{\left|e_{f} B\right|}{\pi}\right) 2\left[4\left|e_{f} B\right| n \delta_{n-1}^{l-1}+\left(\delta_{n}^{l}+\delta_{n-1}^{l-1}\right) m_{f}^{2}+\left(\delta_{n-1}^{l}+\delta_{n}^{l-1}\right)\left(k_{\|}^{2}+k_{\|} \cdot q_{\|}-m_{f}^{2}\right)\right] \\
& =N_{f, n l, \|}\left(q_{\|}, k_{\|}\right)+N_{f, n l, \perp}\left(q_{\|}, k_{\|}\right),
\end{aligned}
$$

where

$$
\begin{gathered}
N_{f, n l, \|}\left(q_{\|}, k_{\|}\right)=(-1)^{n+l}\left(\frac{\left|e_{f} B\right|}{\pi}\right) 2\left[4\left|e_{f} B\right| n \delta_{n-1}^{l-1}+\left(\delta_{n}^{l}+\delta_{n-1}^{l-1}\right) m_{f}^{2}\right], \\
N_{f, n l, \perp}\left(q_{\|}, k_{\|}\right)=(-1)^{n+l}\left(\frac{\left|e_{f} B\right|}{\pi}\right) 2\left[\left(\delta_{n-1}^{l}+\delta_{n}^{l-1}\right)\left(k_{\|}^{2}+k_{\|} \cdot q_{\|}-m_{f}^{2}\right)\right] .
\end{gathered}
$$


Therefore the expressions for $\rho_{\|, \perp}^{q \bar{q}}\left(q_{\|}\right)$will be the same as Eq. (44) except for the fact that $N_{f, n l}(q, k)$ in Eq. (C12) will be replaced by $N_{f, n l, \|}(q, k)$ or $N_{f, n l, \perp}(q, k)$, which are given in Eqs. (92) and (93).

The longitudinal and transverse spectral functions for leptonic case $\rho_{\|, \perp}^{\overline{l l}}\left(q_{\|}\right)$can be obtained from $\rho_{\|, \perp}^{q \bar{q}}\left(q_{\|}\right)$by replacing $T \rightarrow 0, N_{c} \rightarrow 1$, and $\sum_{f} e_{f}^{2} \rightarrow e^{2}$. Considering the dileptons with physical momenta $\left(q^{2}>0, q^{0}>0\right)$, only the Unitary-I cut contributes so that we get

$$
\rho_{\|, \perp}^{\bar{l}}\left(q_{\|}\right)=\sum_{n=0}^{\infty} \sum_{l=(n-1)}^{(n+1)} \frac{\Theta\left(q^{0}-\sqrt{q_{z}^{2}+\left(m_{l}+m_{n}\right)^{2}}\right)}{4 \lambda^{1 / 2}\left(q_{\|}^{2}, m_{l}^{2}, m_{n}^{2}\right)} \sum_{\tilde{k}_{z} \in \tilde{k}_{z}^{ \pm}}\left[N_{\|, \perp}^{n l}\left(q_{\|}, \tilde{k}_{z}, k^{0}=-\tilde{\omega}_{k}^{l}\right)\right],
$$

where $\quad \tilde{k}_{z}^{ \pm}=\frac{1}{2 q_{\|}^{2}}\left[-y q_{z} \pm\left|q^{0}\right| \lambda^{1 / 2}\left(q_{\|}^{2}, m_{l}^{2}, m_{n}^{2}\right)\right], \quad y=\left(q_{\|}^{2}+m_{l}^{2}-m_{n}^{2}\right), \quad \tilde{\omega}_{k}^{l}=\sqrt{\tilde{k}_{z}^{2}+m_{l}^{2}}, \quad$ and $\quad m_{l}=\sqrt{m^{2}+2 l|e B|}$. $N_{\|, \perp}^{n l}\left(q_{\|}, k_{\|}\right)$in the above equation are obtained from Eqs. (92) and (93) by replacing $e_{f} \rightarrow e$. Substituting $N_{\|, \perp}^{n l}\left(q_{\|}, k_{\|}\right)$in the above equation and performing the sum over $\tilde{k}_{z}$, we get, after some simplifications,

$$
\begin{gathered}
\rho_{\|}^{\bar{l}}\left(q_{\|}\right)=\sum_{n=0}^{\infty} \sum_{l=(n-1)}^{(n+1)} \frac{\Theta\left(q_{\|}^{2}-\left(m_{l}+m_{n}\right)^{2}\right)}{\lambda^{1 / 2}\left(q_{\|}^{2}, m_{l}^{2}, m_{n}^{2}\right)} \frac{|e B|}{\pi}\left[4|e B| n \delta_{n-1}^{l-1}+m^{2}\left(\delta_{n}^{l}+\delta_{n-1}^{l-1}\right)\right], \\
\rho_{\perp}^{\bar{l}}\left(q_{\|}\right)=\sum_{n=0}^{\infty} \sum_{l=(n-1)}^{(n+1)} \frac{\Theta\left(q_{\|}^{2}-\left(m_{l}+m_{n}\right)^{2}\right)}{\lambda^{1 / 2}\left(q_{\|}^{2}, m_{l}^{2}, m_{n}^{2}\right)}\left\{\frac{1}{2}\left(q_{\|}^{2}+m_{l}^{2}-m_{n}^{2}\right)-2 l|e B|\right\} \frac{|e B|}{\pi}\left(\delta_{n}^{l-1}+\delta_{n-1}^{l}\right) .
\end{gathered}
$$

For a consistency check, let us consider the LLL approximation for which we have

$$
\begin{gathered}
\rho_{\|, \mathrm{LLL}}^{\bar{l}}\left(q_{\|}\right)=\frac{|e B|}{\pi} \frac{m^{2}}{q_{\|}^{2}}\left(1-\frac{4 m^{2}}{q_{\|}^{2}}\right)^{-1 / 2} \Theta\left(q_{\|}^{2}-4 m_{l}^{2}\right), \\
\rho_{\perp, \mathrm{LLL}}^{\bar{l} \bar{l}}\left(q_{\|}\right)=0,
\end{gathered}
$$

which agrees with the expression in Ref. [53] where the authors have obtained the leptonic contribution by using the spin sum over leptonic spinors in the presence of the external magnetic field.

The presence of the step functions in Eqs. (95) and (96) restricts the kinematic domain for the nonzero DPR in the presence of the external magnetic field. Let us consider the case when the lepton mass is neglected. In that case, the contribution from the LLL vanishes, and thus the kinematic region for nonzero DPR starts from $q_{\|}^{2}>2|e B|$. It will have the consequence that in the presence of a strong enough external magnetic field, the low-invariant mass dileptons $\left(q_{\|}^{2}<2|e B|\right)$ cannot be produced. We will discuss this in detail in the next section.

\section{RESULTS AND DISCUSSIONS}

The numerical results presented in this work are obtained considering $(2+1)$ flavors of the quarks where the masses of up and down quarks are taken to be zero $\left(m_{u}=m_{d}=0\right)$ and the strange quark mass is $m_{s}=100 \mathrm{MeV}$. It is to be noted that, even at very high temperature $(\sim 250 \mathrm{MeV})$, the quarks might still have the effects from the chiral dynamics due to the crossover behavior of the QCD transition from hadron to the QGP in terms of the temperature dependence in their thermal masses [91]. Similar studies incorporating the temperature dependent quark mass for the calculation of DPR can be found in Refs. [92-94]. In addition to the temperature dependence, the quark mass can also depend on the quark virtuality as discussed in Refs. [95-97]. In fact, the quark mass asymptotically reaches the current quark mass values at higher Euclidean momenta of quarks corresponding to the perturbative regime. Thus the effect of momentum dependence of the quark mass at the high temperature is expected to be small. In this work, we have not considered these complications and used constant quark mass in the numerical calculations. In our approach, the effective fugacities capture all the medium effects and contribute to the screening masses of the quarks and antiquarks through the effective couplings. The lepton mass is also considered as zero $(m=0)$. For convergent results at nonzero external magnetic field cases, we have considered up to 1000 Landau levels in the numerical calculations. However, for taking the $e B \rightarrow 0$ limit numerically in Figs. 5 and 9, up to 10000 Landau levels are considered.

Let us start this section by presenting the variation of Debye mass as a function of temperature in Fig. 3(a). At $e B=0$, the $m_{D}$ for the ideal EOS increases monotonically with the increase in $T$ which is obvious from Eq. (25) in 

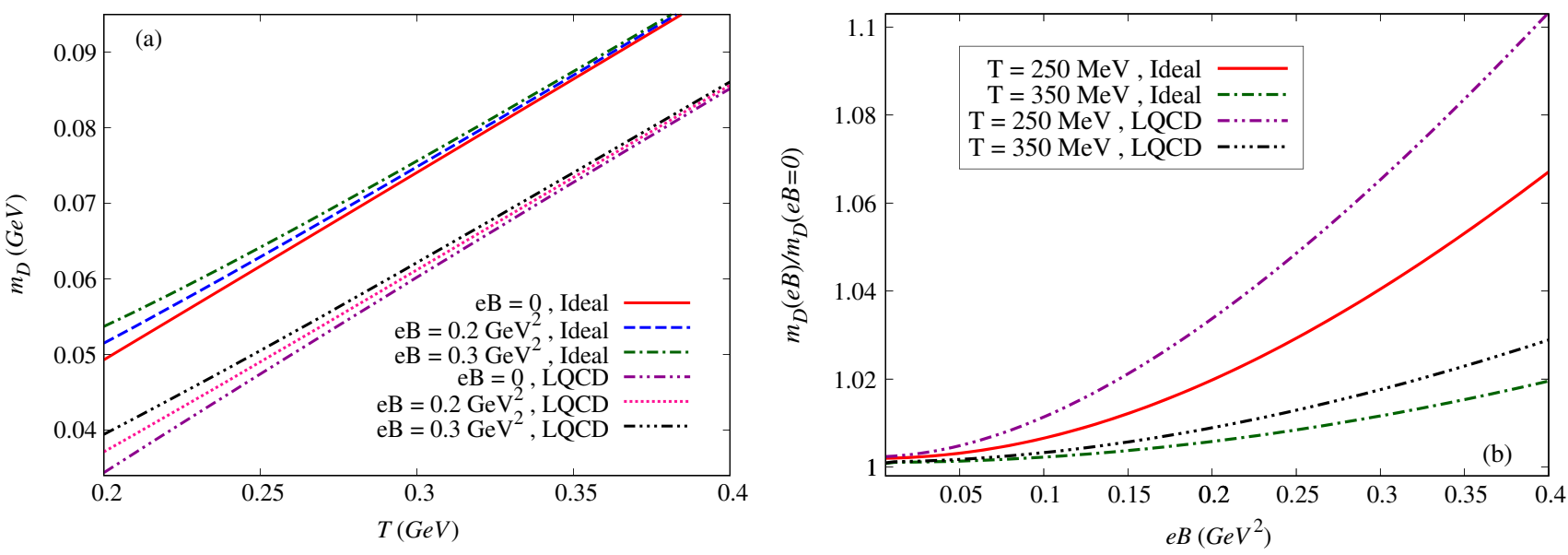

FIG. 3. (a) Debye mass as a function of temperature at different values of external magnetic field $\left(e B=0,0.2\right.$, and $\left.0.3 \mathrm{GeV}^{2}\right)$ for different EOSs. (b) The ratio of Debye mass at finite $e B$ to the Debye mass at zero $e B$ as a function of $e B$ at two different temperatures $(T=250$ and $350 \mathrm{MeV})$ for different EOSs. The "ideal EOS" corresponds to $z_{q}=1$ and "LQCD EOS" corresponds to $z_{q}=z_{q}(T)<1$, which is obtained from Refs. [78,79].

which the thermal distribution functions increase with the increase in temperature. At very high temperature, for which the strange quark mass can be neglected with respect to $T$, we proceed toward the linear relationship of $m_{D}$ with $T$ as given in Eq. (27). For the LQCD EOS $m_{D}$ shows similar variation with $T$ as that of the ideal one. However, the $m_{D}$ at a particular temperature has a lower value for LQCD EOS as compared to the ideal one. This is due to the fact that quasiquarks distribution functions contain the $z_{q}(T)$ that is less than unity. The effect of the strong interactions encoded in $z_{q}(T)$ thus have a significant effect on $m_{D}$. With the increase in $e B, m_{D}$ also increases but by a small amount for the two EOSs. To see the effect of $e B$ more clearly, we have plotted the ratio $m_{D}(e B) / m_{D}(e B=0)$ as a function of $e B$ in Fig. 3(b). We find the effect of $e B$ is more in the case of LQCD EOSs with respect to the ideal EOS. However, for a significant high magnetic field $e B \sim 0.4 \mathrm{GeV}^{2}$, the Debye mass enhances by less than $10 \%$.

The Debye screening mass enhancement in the presence of the external magnetic field is in accordance to the finding in Ref. [98] using lattice QCD simulations and in Refs. $[53,99,100]$ using perturbative calculation. This effect in a certain range of temperatures might be related to the chiral symmetry breaking in terms of magnetic catalysis as argued in Refs. [53,99]. However, to make a concrete statement, we require knowing the role of the external electromagnetic field on plasma relaxation processes in QGP, which is beyond the scope of the present study.

Next we show the results of electromagnetic spectral functions due to the quark loop in the magnetized hot QCD medium. Let us first consider the zero magnetic field case in Fig. 4. We have plotted $\rho^{q \bar{q}}(q)$ as a function of invariant
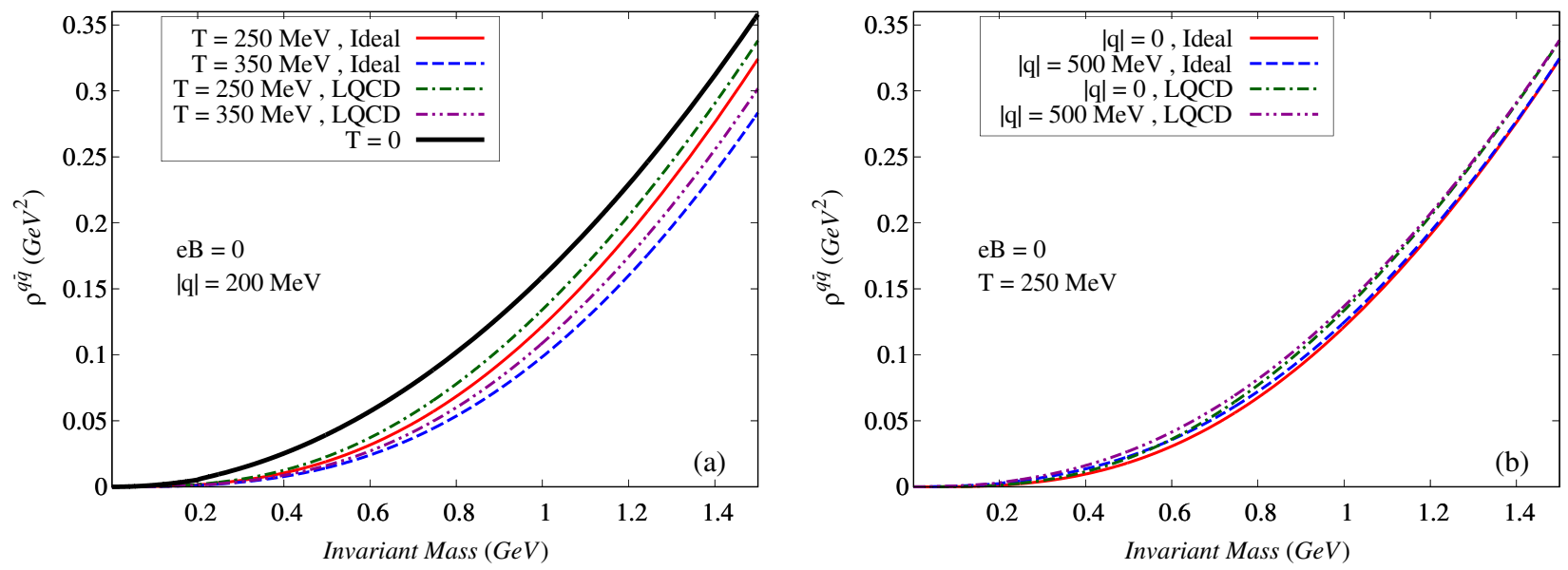

FIG. 4. Electromagnetic spectral function due to the quark loop for different EOSs as a function of the photon invariant mass at the zero external magnetic field: (a) with photon three momentum $\vec{q}=200 \mathrm{MeV}$ at two different temperatures (250 and $350 \mathrm{MeV}$ ), and (b) at constant temperature $(250 \mathrm{MeV})$ and at two different photon three momentum $\vec{q}$ ( 0 and $500 \mathrm{MeV})$. The vacuum spectral function $(T=0)$ is also shown in (a) for comparison. 


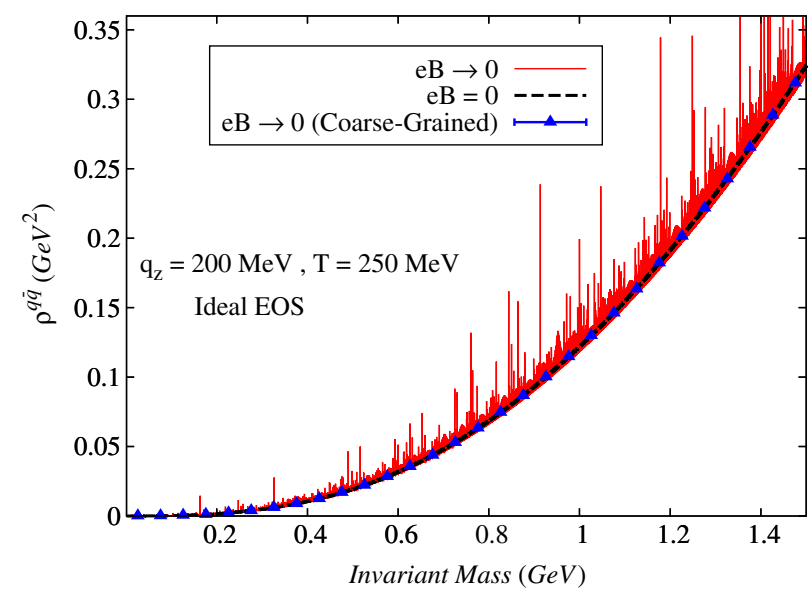

FIG. 5. Electromagnetic spectral function due to the quark loop as a function of the photon invariant mass at $T=250 \mathrm{MeV}, q_{z}=$ $200 \mathrm{MeV}$ for ideal EOS. Results for $e B=0$ have been compared among that of $e B \rightarrow 0$ and the coarse-grained $e B \rightarrow 0$.

mass $\sqrt{q^{2}}$ at $|\vec{q}|=200 \mathrm{MeV}$ and at two different temperatures (250 and $350 \mathrm{MeV}$ ) for different EOSs in Fig. 4(a). The zero temperature case is also shown for comparison. As we have discussed in the previous section, in the absence of $e B$, only the Unitary-I cut contributes to the spectral function that starts from $q^{2} \geq 4 m_{f}^{2}$. Since we have taken $m_{u}=m_{d}=0$, the spectral function is nonzero at $q^{2}>0$. With the increase in invariant mass, $\rho^{q \bar{q}}(q)$ increases monotonically due to the increase in the availability of the phase space. Additionally, with the increase in $T$, the spectral function decreases. It can be understood from Eq. (35) where the quark distribution functions increase with an increase in $T$ in turn reduce the overall factor $\tanh \left(\frac{\beta q^{0}}{2}\right)\left\{1-f\left(\omega_{k}^{f}\right)-f\left(\omega_{p}^{f}\right)+2 f\left(\omega_{k}^{f}\right) f\left(\omega_{p}^{f}\right)\right\}$ in the Unitary cut restricting the phase space availability of the quarks. The effect of inclusion of effective fugacity in the quasiquark distribution function has an opposite effect

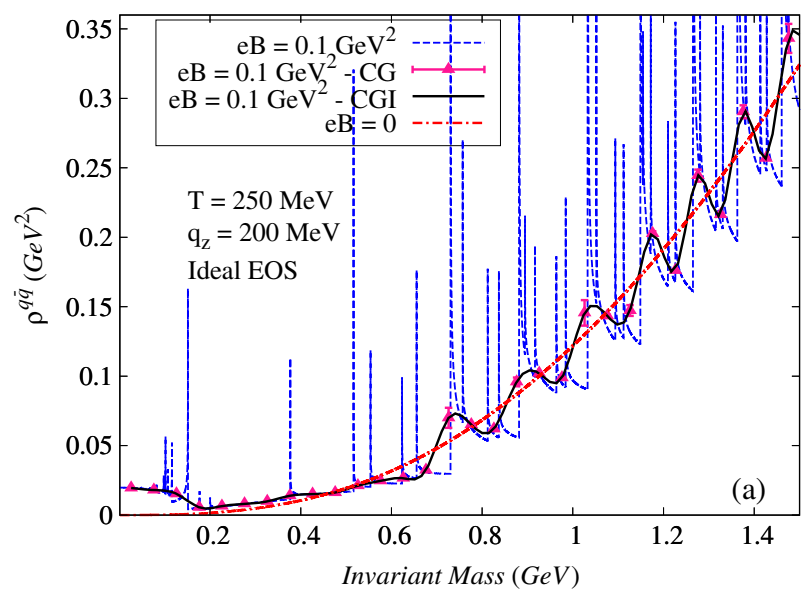

with respect to the increase in temperature. Because of $z_{q}(T)<1$, the phase space is more in the LQCD EOS case as compared to the ideal one. In Fig. 4(b) we have shown $\rho^{q \bar{q}}(q)$ as a function of invariant mass at a constant temperature $T=250 \mathrm{MeV}$ and at two different values of photon three momentum $(|\vec{q}|=0$ and $500 \mathrm{MeV})$ for the different EOSs. It is observed that the effect of an increase of $|\vec{q}|$ on $\rho^{q \bar{q}}(q)$ is very small and it only affects at the low invariant mass region.

We now turn on the external magnetic field. For a consistency check, we have first taken the $e B \rightarrow 0$ limit numerically to Eq. (44) and compared with $\rho^{q \bar{q}}$ calculated from Eq. (35) in Fig. 5. The results are presented for ideal EOS at $T=250 \mathrm{MeV}$ and $q_{z}=200 \mathrm{MeV}$. We found a large number of spikes infinitesimally spaced from each other covering the whole invariant mass axis. The $e B=0$ graph is analytic having no such spikes, and interestingly it goes on average through the $e B \rightarrow 0$ graph. The appearance of these spikes are due to the "threshold singularities" in each Landau level as can be seen from Eq. (44), where the Källén function in the denominator blows up in each threshold defined in terms of the step functions therein. This type of threshold singularities are observed in other works as well $[53,101]$. In order to extract finite and physical results for the spectral function we have introduced the concept of Ehrenfest's coarse graining [102,103] in which we have discretized the whole invariant mass region in small bins followed by performing bin averages. Thus, in other words, $\rho^{q \bar{q}}\left(q_{\|}\right)$at a given $\sqrt{q_{\|}^{2}}$ is approximated by its average over the neighborhood around that point. We calculated the coarse-grained (CG) spectral functions and show them in Fig 5. It can be noticed that the CG $\rho^{q \bar{q}}(q)$ for $e B \rightarrow 0$ exactly reproduce the $e B=0$ case.

We now increase the $e B$ and show the spectral function due to the quark loop for finite values of magnetic field $\left(e B=0.1\right.$ and $\left.0.2 \mathrm{GeV}^{2}\right)$ in Figs. 6(a) and 6(b),

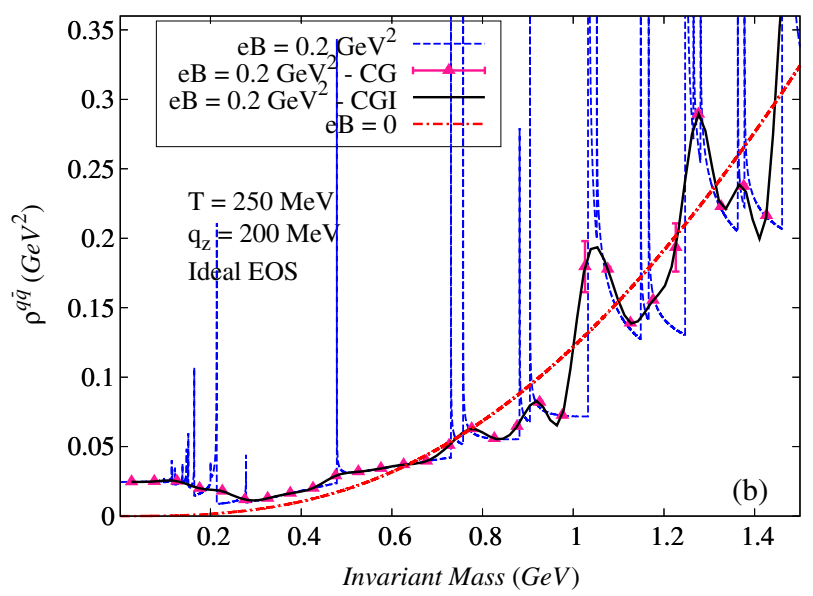

FIG. 6. Electromagnetic spectral function due to the quark loop as a function of the photon invariant mass at $T=250 \mathrm{MeV}$, $q_{z}=200 \mathrm{MeV}$ for ideal EOS. Results for $e B=0$ have been presented along with (a) $e B=0.1$ and (b) $e B=0.2 \mathrm{GeV}^{2}$. The coarsegrained (CG) as well as coarse-grained interpolated (CGI) results for nonzero $e B$ are also shown for comparison. 
respectively. The EOS, $T$, and $q_{z}$ are taken the same as Fig. 5. The $e B=0$ case is also shown for comparison. As discussed in the previous section, in this case the Unitary-I cut begins from $q_{\|}^{2} \geq 4 m_{f}^{2}$. Since we have taken $m_{u}=m_{d}=0$, the threshold of the Unitary-I cut for these two massless flavors is $q_{\|}^{2} \geq 0$, whereas the same is $q_{\|}^{2} \geq 4 m_{s}^{2}$ for the strange quark. It is to be noted from the discussions in Appendix D that the Unitary-I cut threshold comes from the LLL. However, for the massless quark flavors the contribution to the spectral function from LLL vanishes as is evident from Eq. (C12). Hence for the massless quark flavors, the threshold of the Unitary-I cut will be $q_{\|}^{2}>2\left|e_{f} B\right|$, which comes from the next to LLL. Thus when all the quark flavors are summed up, the threshold of the Unitary-I cut will become $q_{\|}^{2}>\min \left(2 \times \frac{1}{3}|e B|, 4 m_{s}^{2}\right)$. The spectral functions suffer lots of threshold singularities and are now separated from each other by the finite value of the invariant mass (unlike Fig. 5 where they were infinitesimally spaced). With the increase in $e B$ the spacing among these spikes increases as can be seen from Eq. (44). Moreover, the spectral functions for nonzero $e B$ perform oscillatory behaviors about the $e B=0$ graph at a higher invariant mass region. This is more clearly visible when we plot the CG spectral functions. The CG spectral functions at the discrete bin points are used to obtain an interpolated graph that we call the CG interpolated (CGI) spectral function. The oscillatory behaviors of the spectral function can be best observed in the CGI graphs. This oscillation frequency is more at the lower $e B\left(0.1 \mathrm{GeV}^{2}\right)$ as compared to the higher $e B\left(0.2 \mathrm{GeV}^{2}\right)$ but the amplitude of oscillation is just the opposite (low for lower $e B$ and high for higher $e B$ ). This is consistent with Fig. 4 where at a limiting value of $e B \rightarrow 0$, the oscillation frequency becomes infinite and the amplitude becomes zero reproducing the $e B=0$ graph. It is important to mention that, in the case of nonzero $e B$, the Landau cuts also contribute (as discussed in the previous section) and, in fact, the lower invariant mass region is dominated by these Landau terms that will be clear in the next paragraph.

In order to see the significance of the Landau terms, we have compared the contribution to $\rho^{q \bar{q}}(q)$ arising from the Unitary and Landau cuts separately in Fig. 7. The result is obtained for ideal EOS with $T=250 \mathrm{MeV}$, $q_{z}=200 \mathrm{MeV}$, and $e B=0.3 \mathrm{GeV}^{2}$. As can be seen from the figure, the low invariant mass region is dominated by the Landau cut contribution where the high invariant mass region is dominated by the Unitary cut contribution. Moreover, the Landau cut is extended only up to $\sqrt{q_{\|}^{2}} \leq \sqrt{2\left|Q_{f}\right| e B}$ with the maximum $Q_{f}=2 / 3$ for the up quark as can be understood from the previous section. This threshold of the Landau cut is shown by the black horizontal line in the figure. As discussed earlier, the Unitary cut threshold in this case is $\sqrt{q_{\|}^{2}} \geq$ $\min \left(\sqrt{2 \times \frac{1}{3}|e B|}, 2 m_{s}\right)=2 m_{s} \quad\left(\right.$ since $\quad e B=0.3 \mathrm{GeV}^{2}$,

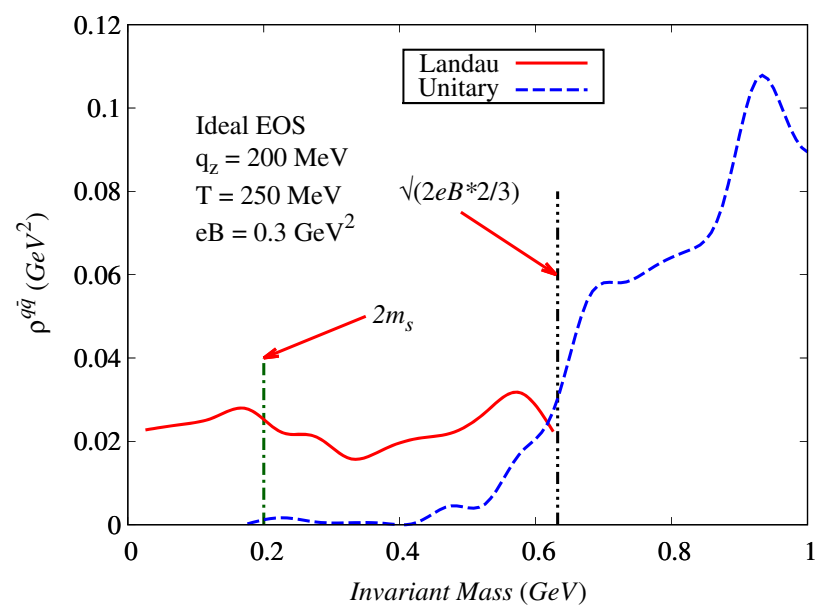

FIG. 7. The Landau and Unitary contributions to the coarsegrained interpolated electromagnetic spectral function due to the quark loop as a function of the photon invariant mass at $T=250 \mathrm{MeV}, q_{z}=200 \mathrm{MeV}$, and $e B=0.3 \mathrm{GeV}^{2}$ for ideal EOS. The vertical black line correspond to the Landau-cut threshold which is $\sqrt{2 e B \times 2 / 3}$ (due to the up quark). The vertical green lines correspond to the Unitary-cut threshold, which is $2 m_{s}$ (due to the strange quark).

$m_{s}=100 \mathrm{MeV}$ ), which is shown by the green vertical line in the figure.

In Fig. 8(a) we have shown CGI $\rho^{q \bar{q}}(q)$ as a function of the invariant mass for three different values of $e B(0,0.1$, and $0.3 \mathrm{GeV}^{2}$ ) at $T=250 \mathrm{MeV}$ and $q_{z}=200 \mathrm{MeV}$ for ideal EOS. We find significance enhancement of $\rho^{q \bar{q}}(q)$ with the increase in $e B$ at the low invariant mass regions where as at the higher values of invariant mass, the spectral function is oscillatory about the $e B=0$ graph. Analogous results for the LQCD EOS is presented in Fig. 8(b).

Let us now turn our attention to the results of the electromagnetic spectral function due to lepton loop $\rho^{\bar{l}}=\rho_{\|}^{\bar{l}}+\rho_{\perp}^{\bar{l}}$. For a consistency check, we have first taken the $e B \rightarrow 0$ limit numerically from Eqs. (95) and (96) and compared with $\rho^{\bar{l}}$ calculated from Eq. (83) in Fig. 9. The results are presented for $q_{z}=200 \mathrm{MeV}$. Analogous to $\rho^{q \bar{q}}$, we found a large number of infinitesimally spaced spikes covering the whole invariant mass axis. The $e B=0$ graph goes on average through the $e B \rightarrow 0$ graph. The appearance of these spikes are due to the threshold singularities in each Landau level as can be seen from Eqs. (95) and (96) where the Källén lambda function in the denominators blows up in each threshold defined in terms of the step functions therein. The CG spectral function in this case also reproduces the $e B=0$ graph exactly. It is to be noted that in this case we do not have any Landau cut contribution since the temperature is zero.

Next, in Figs. 10(a) and 10(b), $\rho^{l \bar{l}}$ is shown at $e B=0.1$ and $02 \mathrm{GeV}^{2}$, respectively, for $q_{z}=200 \mathrm{MeV}$. In this case the threshold of the Unitary cut begins from $\sqrt{q_{\|}}>\sqrt{2 e B}$ as discussed in the last paragraph of the previous section. 

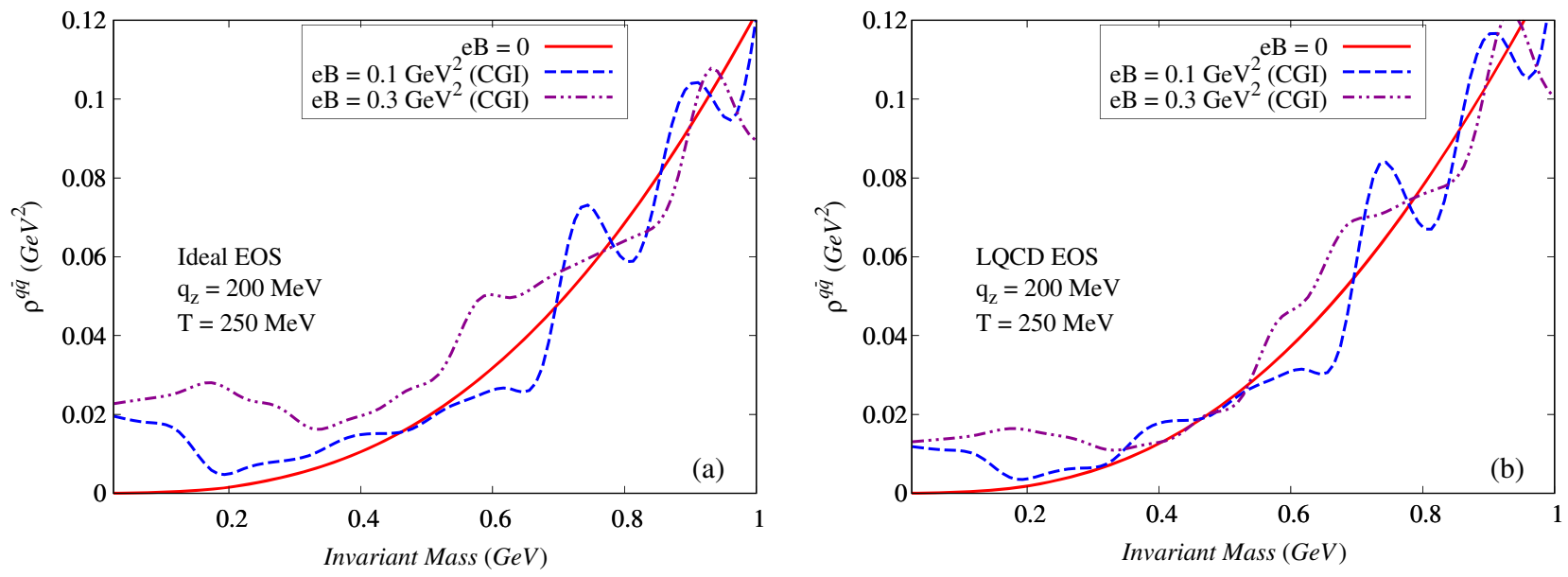

FIG. 8. The coarse-grained interpolated electromagnetic spectral function due to the quark loop as a function of photon invariant mass at $T=250 \mathrm{MeV}, q_{z}=200 \mathrm{MeV}$, and three different vacules of $e B\left(0,0.1\right.$, and $\left.0.3 \mathrm{GeV}^{2}\right)$ for (a) ideal and (b) LQCD EOS.

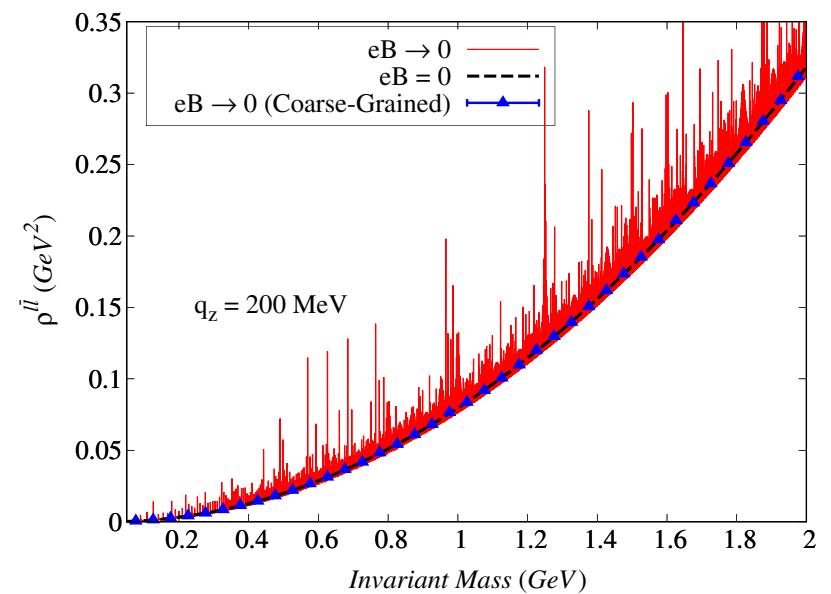

FIG. 9. Electromagnetic spectral function due to the lepton loop as a function of the photon invariant mass at $q_{z}=200 \mathrm{MeV}$. Results for $e B=0$ have been compared among that of $e B \rightarrow 0$ and the coarse-grained $e B \rightarrow 0$.

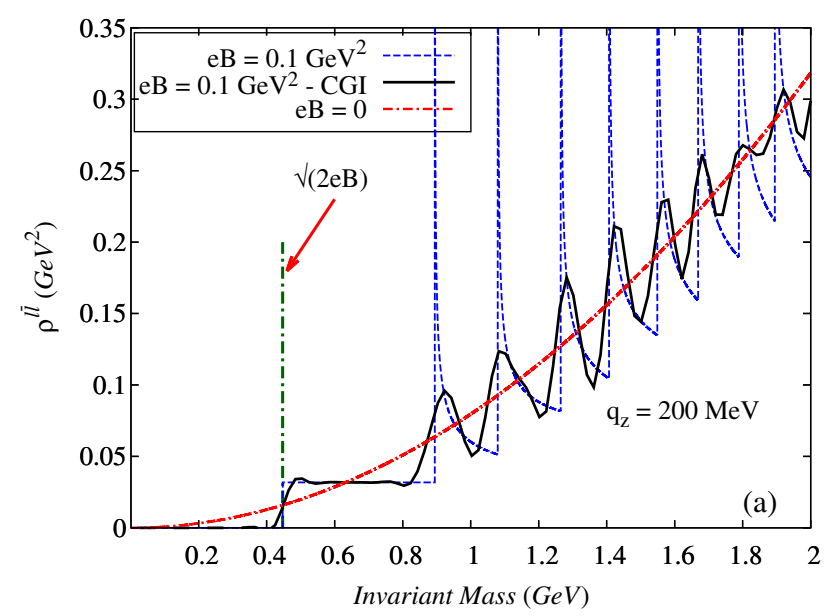

The spectral function shows oscillatory behavior about the $e B=0$ graph at nonzero $e B$ and the oscillation frequency (amplitude) decreases (increases) with the increase of the external magnetic field.

Next, we investigate how the DPR from the hot QCD medium gets modified due to the external magnetic field as well as the EOS effects. Let us first consider the $e B=0$ case. We have plotted the ratio $\mathrm{DPR}_{\mathrm{Born}} / \mathrm{DPR}$ as a function of invariant mass of the dilepton in Fig. 11 where $\mathrm{DPR}_{\mathrm{Born}}$ is the Born rate for the dilepton production given by [51] (for lepton mass $m=0$ )

$$
\begin{aligned}
\mathrm{DPR}_{\mathrm{Born}}= & \frac{\alpha^{2} N_{c} T}{6 \pi^{4}|\vec{q}|}\left(\frac{1}{e^{\beta q^{0}}-1}\right) \sum_{f} Q_{f}^{2}\left(1+\frac{2 m_{f}^{2}}{q^{2}}\right) \\
& \times \ln \left[\frac{\cosh \left(\frac{q^{0}+R_{f}|\vec{q}|}{4 T}\right)}{\cosh \left(\frac{q^{0}-R_{f}|\vec{q}|}{4 T}\right)}\right] \Theta\left(q^{2}-4 m_{f}^{2}\right),
\end{aligned}
$$

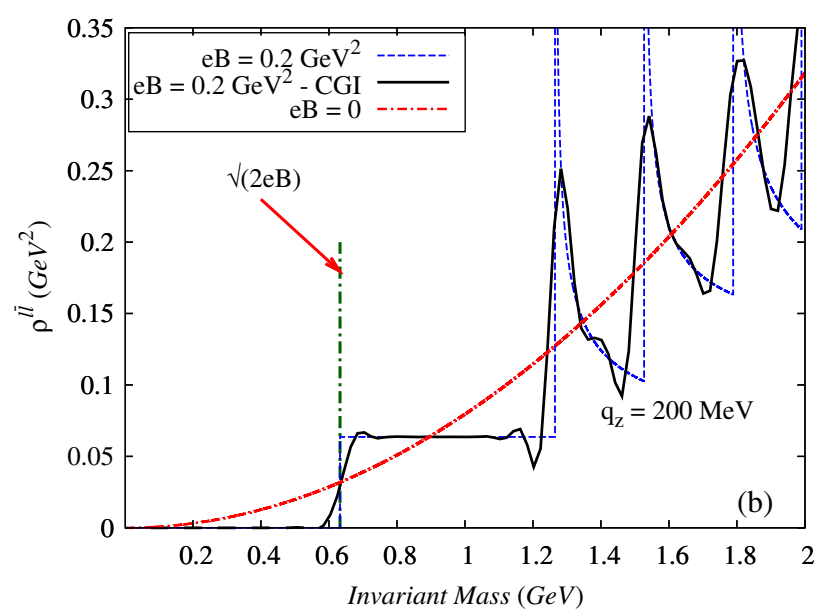

FIG. 10. Electromagnetic spectral function due to the lepton loop as a function of the photon invariant mass at $q_{z}=200 \mathrm{MeV}$. Results for $e B=0$ have been presented along with (a) $e B=0.1$ and (b) $e B=0.2 \mathrm{GeV}^{2}$. The CG as well as CGI results for nonzero $e B$ are also shown for comparison. The vertical green lines correspond to the Unitary cut threshold $\sqrt{q_{\|}^{2}}=\sqrt{2 e B}$ for the massless leptons in the
presence of the external magnetic field. 


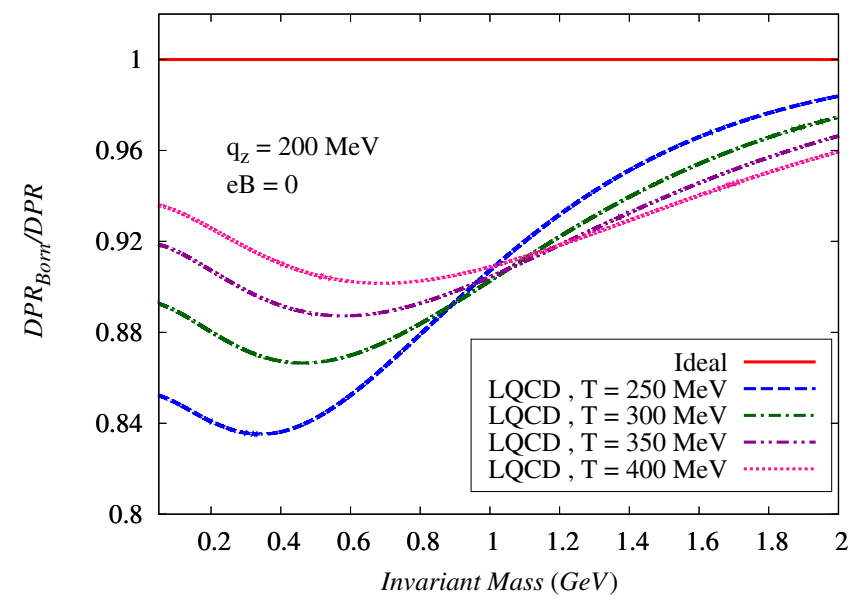

FIG. 11. The ratio of the Born rate to DPR as a function of the dilepton invariant mass at the zero external magnetic field for $q_{z}=200 \mathrm{MeV}$. Results for the ideal EOS has been compared among the LQCD EOS at four different temperatures $(250,300$, 350, and $400 \mathrm{MeV}$ ).

where $R_{f}=\left(1-\frac{4 m_{f}^{2}}{q^{2}}\right)^{1 / 2}$. The results are obtained at four different temperatures $(250,300,350$, and $400 \mathrm{MeV})$ and at $q_{z}=200 \mathrm{MeV}$ for different EOSs. As can be seen from the graph, our result for the ideal EOS exactly matches with the Born rate so that the ratio is unity and it is independent of temperature. For the other two EOSs, we find significant enhancement of the DPR with respect to the Born rate. The enhancement is more at a higher temperature, and as we increase $T$, we move toward the unity. Even at temperature $T=250 \mathrm{MeV}$, we find $\simeq 15 \%$ enhancement of the DPR with respect to the Born rate. At lower temperature, the enhancement will be even more.

In the presence of the nonzero external magnetic field, the DPR is analyzed for the following two cases separately:

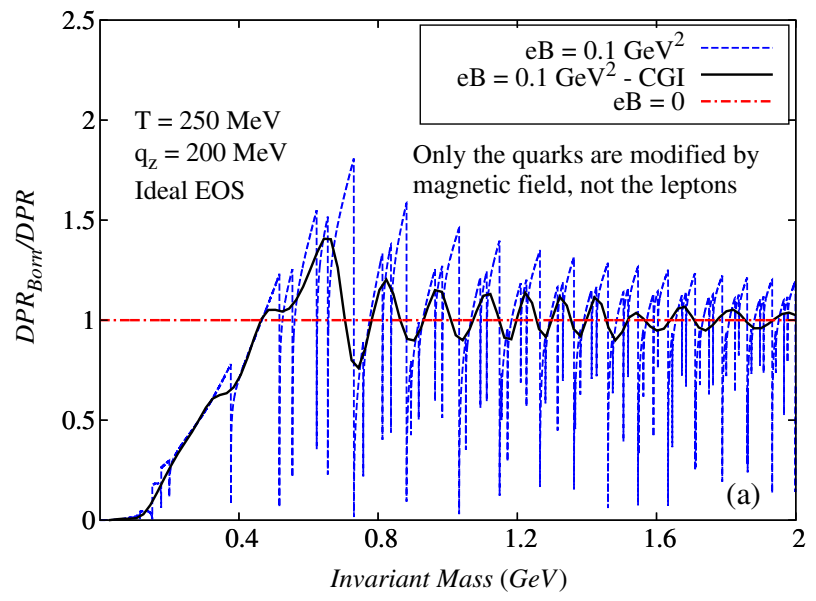

(1) Only the quarks are affected by the external magnetic field, not the dileptons, and (2) both the quarks as well as the dileptons are affected by the external magnetic field.

Now for a consistency check, we have first considered the LLL approximation and observed that the results of Ref. [53] could be reproduced by taking the ideal EOS and finite masses of quark flavors. In the LLL approximation, the DPR vanishes for massless quark flavors as evident from Eq. (C12) and also shown in Ref. [53].

In Figs. 12(a) and 12(b), we have plotted DPR $\mathrm{Born} /$ DPR as a function of the dilepton invariant mass at $e B=0.1 \mathrm{GeV}^{2}, q_{z}=200 \mathrm{MeV}, T=250 \mathrm{MeV}$ with ideal EOS for case (1) and case (2), respectively. The $e B=0$ graph is also shown for comparison. For both the cases, we find significant enhancement of the low invariant mass dilepton production, which is due to the appearance of the Landau cuts in the presence of $e B$ which was absent in the $e B=0$ case. However, at the higher invariant mass region, the DPR shows oscillatory behavior about the $e B=0$ graph. The oscillation amplitude is more for case (2). It is to be noted that, in case (2), there is no dilepton production at $\sqrt{q_{\|}^{2}}<\sqrt{2 e B}$, which is due to the threshold of $\rho^{\bar{l}}$ as shown in Fig. 10.

We conclude this section by finally presenting the CGI $\mathrm{DPR}_{\mathrm{Born}} / \mathrm{DPR}$ as a function of invariant mass of the dileptons for three different values of $e B(0,0.1$, and $0.3 \mathrm{GeV}^{2}$ ) in Fig. 13. Results are obtained at $T=$ $250 \mathrm{MeV}$ and $q_{z}=200 \mathrm{MeV}$. The left panel corresponds to ideal EOS, whereas the right panel is for LQCD EOS. The top panel corresponds to the results for case (1), whereas the bottom panel depicts the corresponding results for case (2). With the increase in $e B$, the DPR enhances in the low invariant mass region for both the cases. However, because of the kinematic threshold of the dilepton

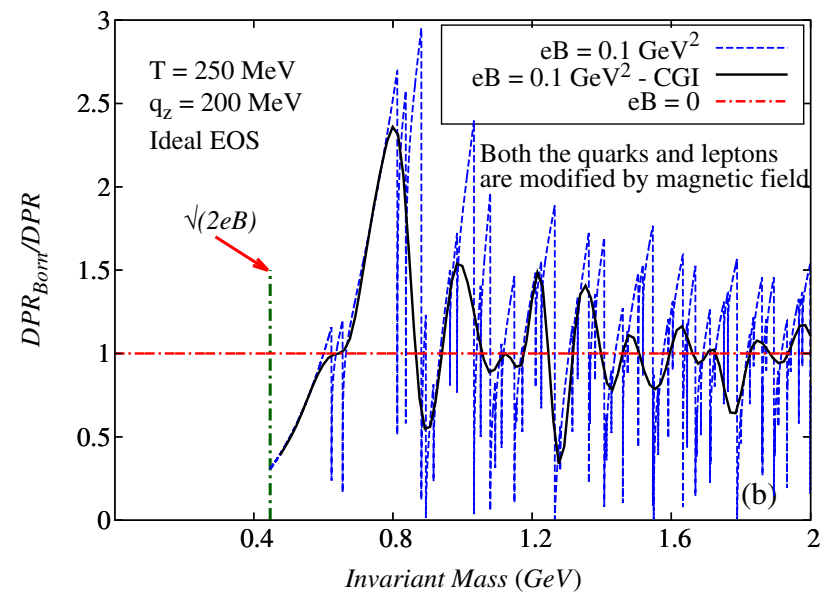

FIG. 12. The ratio of the Born rate to DPR as a function of the dilepton invariant mass at $T=250 \mathrm{MeV}, q_{z}=200 \mathrm{MeV}$, and at two different values of $e B\left(0\right.$ and $\left.0.1 \mathrm{GeV}^{2}\right)$ for the ideal EOS. Coarse-grained interpolated results are also shown for the nonzero $e B$ case. (a) Case where the quarks are modified by the magnetic field, not the leptons, and (b) case when both the quarks as well as the lepton are modified by the magnetic field. The vertical green line in (b) corresponds to the DPR threshold $\sqrt{q_{\|}^{2}}=\sqrt{2 e B}$. 

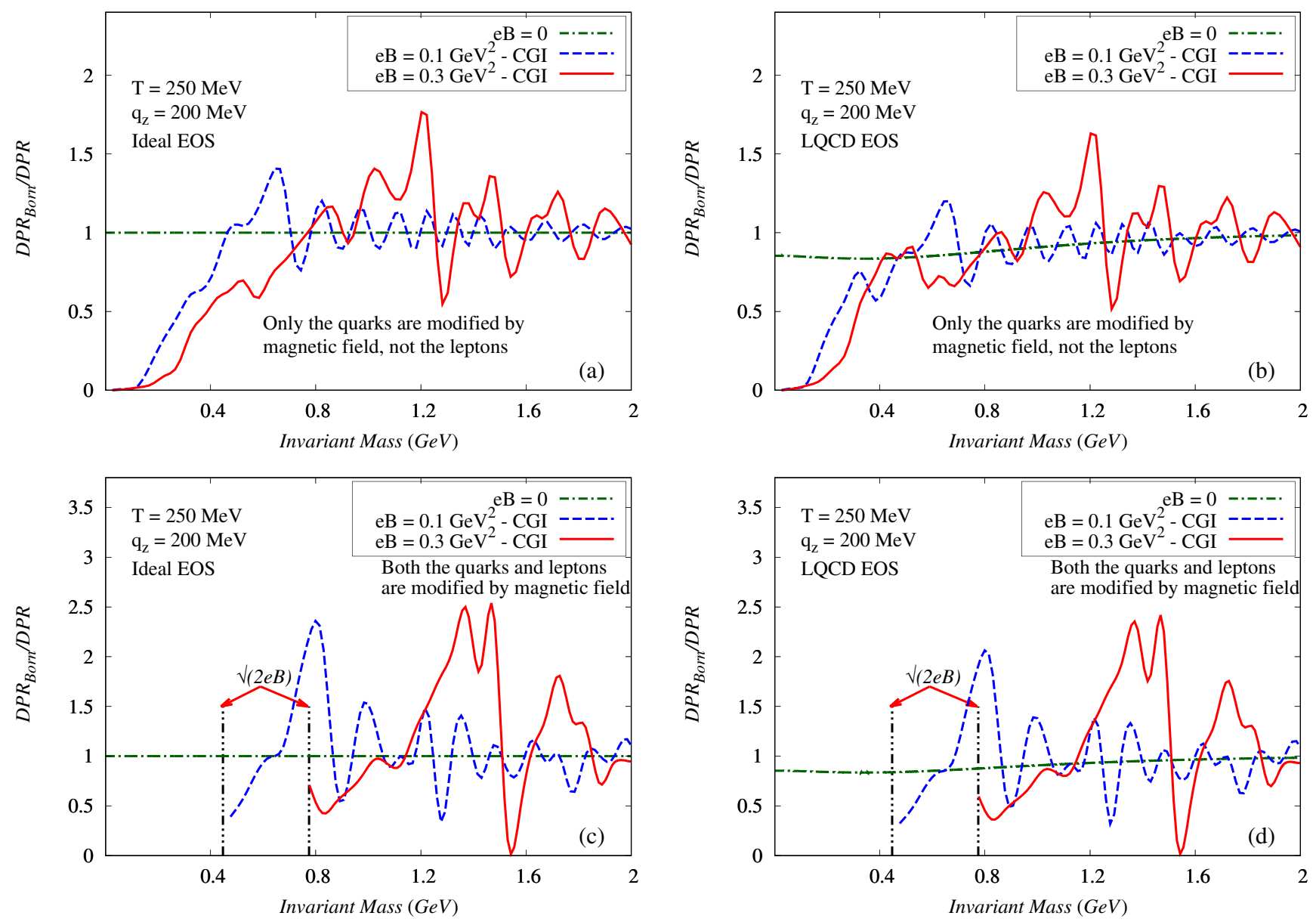

FIG. 13. The ratio of the Born rate to the coarse-grained interpolated DPR as a function of the dilepton invariant mass at $T=250 \mathrm{MeV}, q_{z}=200 \mathrm{MeV}$, and at three different values of $e B\left(0,0.1\right.$, and $\left.0.3 \mathrm{GeV}^{2}\right)$ for (left panel) ideal and (right panel) LQCD EOSs. The top panel shows the case where the quarks are modified by the magnetic field, not the leptons, and the bottom panel shows the case where both the quarks as well as the lepton are modified by the magnetic field. The vertical green lines in the bottom panel correspond to the DPR threshold $\sqrt{q_{\|}^{2}}=\sqrt{2 e B}$.

production at finite $e B$, the dilepton production starts from the invariant mass $\sqrt{q_{\|}^{2}}>\sqrt{2 e B}$. At higher invariant mass regions, the ratio oscillates about the $e B=0$ graph, and thus we get both the enhancement and suppression of dilepton production at different invariant masses.

\section{SUMMARY AND CONCLUSIONS}

In summary, we have studied the electromagnetic spectral function and dilepton production rate in the presence of both finite temperature and external magnetic field in a QCD medium. The photon polarization tensor at finite temperature is calculated using the RTF of TFT where the thermal distribution functions of loop particles (quarks/ antiquarks) are modified in terms of their effective fugacities. The effective fugacity in the (quasi)quark distribution function encodes the hot QCD medium effect and is consistent with the realistic QCD EOSs like LQCD
EOS. The effect of the external magnetic field is introduced through the modification of the quark propagator in terms of the Schwinger proper time propagator including all the Landau levels without any approximation on the strength of the external magnetic field. The Debye screening mass that has been obtained from the real part of the thermal photon self-energy under the external magnetic field turns out to be exactly the same as what could be obtained from linearized semiclassical transport theory. The imaginary part of the thermal photon self-energy function has been obtained across the Unitary and Landau cuts. The Unitary cuts are already present in the case of the zero external magnetic field, whereas the Landau cuts appeared purely due to the external magnetic field. Thereafter the analytical structure of the spectral function is studied and the magnetic field dependent thresholds of the Unitary and Landau cuts are obtained, which are different from the $e B=0$ case. While calculating the DPR from the thermal QGP medium, we 
have expressed it in terms of the product of electromagnetic spectral functions due to quark and lepton loops. This formalism allows us to introduce the external magnetic field in terms of the modification of quark and lepton propagators by the Schwinger proper time one so that the knowledge of spin sum over leptonic spinor in the presence of the magnetic field is not required. We have observed spikelike structure in both electromagnetic spectral functions due to quark and lepton loops that arise because of the threshold singularity in each Landau level. The numerical results in the limit $e B \rightarrow 0$ exactly reproduce the $e B=0$ result when the Ehrenfest's coarse-graining technique is used, which smears out the threshold singularities.

In conclusion, the significant enhancement of the electromagnetic Debye screening mass with the increase in both the magnetic field and temperature has been observed, whereas the inclusion of hot QCD medium effects decreases the magnitude of the Debye mass. The electromagnetic spectral function is seen to decrease with the increase in temperature, and it also has nontrivial dependence on the external magnetic field. The spectral function in the low invariant mass region (dominated by the Landau term) increases with the increase in $e B$, whereas the high invariant mass region (dominated by the Unitary term) shows an oscillatory behavior about the $e B=0$ values. The oscillation frequency (amplitude) decreases (increases) with the increase in $e B$. The spectral function due to the lepton loop shows identical behavior except for the fact that in this case, the Landau cut does not appear. Thus the spectral function for the lepton loop containing only the Unitary term has a particular threshold in the invariant mass $(>\sqrt{2 e B})$. This in turn restricts the kinematics for the low invariant mass dilepton production. A significant enhancement of the low invariant mass dilepton yield with respect to the Born rate has been observed due to the appearance of the Landau terms. On the other hand, in the high invariant mass region, an oscillation about the Born rate is noticed where the dilepton yield may enhance or suppress depending on the invariant mass of the dilepton. Finally, our results are consistent with the predictions of other approaches (in limiting cases, whenever possible/applicable).
The immediate further extension of the work could be the inclusion of dissipative effects in the hot medium and couple the analysis with the (magneto)hydrodynamic framework in order to relate the relevant quantities to the experimentally realizable observables.

\section{ACKNOWLEDGMENTS}

S. G. acknowledges Professor Sourav Sarkar for the help in the field theoretical calculations. V.C. acknowledges SERB, Government of India for Early Career Research Grant (ECRA/2016/000683) and INSA-Department of Science and Technology, Government of India for INSPIRE Faculty Grant (IFA-13/PH-55). S. G. also acknowledges the Indian Institute of Technology Gandhinagar for the postdoctoral fellowship. We are highly grateful to the people of India for their generous support for the research in fundamental sciences.

\section{APPENDIX A: USEFUL IDENTITIES}

Using the orthogonality properties of the generalized Laguerre polynomials, one can derive the following identities:

$$
\begin{gathered}
\int \frac{d^{2} k_{\perp}}{(2 \pi)^{2}} e^{-2 \alpha_{k}} k_{\perp}^{2} L_{l-1}^{1}\left(2 \alpha_{k}\right) L_{n-1}^{1}\left(2 \alpha_{k}\right)=-\frac{\left(e_{f} B\right)^{2}}{16 \pi} n \delta_{n-1}^{l-1}, \\
\int \frac{d^{2} k_{\perp}}{(2 \pi)^{2}} e^{-2 \alpha_{k}} L_{l}\left(2 \alpha_{k}\right) L_{n}\left(2 \alpha_{k}\right)=\frac{\left|e_{f} B\right|}{8 \pi} \delta_{n}^{l},
\end{gathered}
$$

where $\alpha_{k}=-k_{\perp}^{2} /\left|e_{f} B\right|$.

\section{APPENDIX B: CALCULATION OF $\operatorname{Re} \bar{\Pi}^{00}\left(q^{0}=0, \vec{q} \rightarrow \overrightarrow{0}\right)$ FOR $B \neq 0$}

In this Appendix, we will sketch how to obtain Eq. (29). For this first we put $q_{\perp}=0$ in Eq. (20) and consider the 00 component

$$
\begin{aligned}
\operatorname{Re} \bar{\Pi}^{00}\left(q_{\|}\right)= & \sum_{f} e_{f}^{2} N_{c} \sum_{l=0}^{\infty} \sum_{n=0}^{\infty} \int \frac{d^{3} k}{(2 \pi)^{3}} \mathcal{P}\left[\frac{f\left(\omega_{k, l}^{f}\right)}{2 \omega_{k, l}^{f}}\left\{\frac{N_{f, n l}^{00}\left(k^{0}=-\omega_{k, l}^{f}\right)}{\left(q^{0}-\omega_{k, l}^{f}\right)^{2}-\left(\omega_{p, n}^{f}\right)^{2}}+\frac{N_{f, n l}^{00}\left(k^{0}=\omega_{k, l}^{f}\right)}{\left(q^{0}+\omega_{k, l}^{f}\right)^{2}-\left(\omega_{p, n}^{f}\right)^{2}}\right\}\right. \\
& \left.+\frac{f\left(\omega_{p, n}^{f}\right)}{2 \omega_{p, n}^{f}}\left\{\frac{N_{f, n l}^{00}\left(k^{0}=-q^{0}-\omega_{p, n}^{f}\right)}{\left(q^{0}+\omega_{p, n}^{f}\right)^{2}-\left(\omega_{k, l}^{f}\right)^{2}}+\frac{N_{f, n l}^{00}\left(k^{0}=-q^{0}+\omega_{p, n}^{f}\right)}{\left(q^{0}-\omega_{p, n}^{f}\right)^{2}-\left(\omega_{k, l}^{f}\right)^{2}}\right\}\right]+\operatorname{Re} \Pi_{v a c}^{00}\left(q_{\|}\right)+\operatorname{Re} \Pi_{\mathrm{B}}^{00}\left(q_{\|}, B\right) .
\end{aligned}
$$

Calculating the trace over Dirac matrices in Eq. (23), we get

$$
\begin{aligned}
N_{f, n l}^{00}= & (-1)^{n+l} e^{-2 \alpha_{k}} 8\left[-8 L_{l-1}^{1}\left(2 \alpha_{k}\right) L_{n-1}^{1}\left(2 \alpha_{k}\right) k_{\perp}^{2}+\left\{L_{l}\left(2 \alpha_{k}\right) L_{n}\left(2 \alpha_{k}\right)+L_{l-1}\left(2 \alpha_{k}\right) L_{n-1}\left(2 \alpha_{k}\right)\right\}\right. \\
& \left.\times\left(m_{f}^{2}+k_{0}^{2}+k_{z}^{2}+k^{0} q^{0}+k_{z} q_{z}\right)\right] .
\end{aligned}
$$


Substituting the above equation into Eq. (B1) and performing the $d^{2} k_{\perp}$ integral using the identities given in Appendix A, we get after putting $q^{0}=0$

$$
\begin{aligned}
\operatorname{Re} \bar{\Pi}^{00}\left(q_{z}\right)= & \sum_{f} e_{f}^{2} N_{c} \sum_{l=0}^{\infty} \sum_{n=0}^{\infty} \int_{-\infty}^{\infty} \frac{d k_{z}}{2 \pi} \mathcal{P}\left[\frac{f\left(\omega_{k, l}^{f}\right)}{2 \omega_{k, l}^{f}}\left\{\frac{\tilde{N}_{f, n l}^{00}\left(k^{0}=-\omega_{k, l}^{f}\right)+\tilde{N}_{f, n l}^{00}\left(k^{0}=\omega_{k, l}^{f}\right)}{\left(\omega_{k, l}^{f}\right)^{2}-\left(\omega_{p, n}^{f}\right)^{2}}\right\}\right. \\
& \left.+\frac{f\left(\omega_{p, n}^{f}\right)}{2 \omega_{p, n}^{f}}\left\{\frac{\tilde{N}_{f, n l}^{00}\left(k^{0}=-\omega_{p, n}^{f}\right)+\tilde{N}_{f, n l}^{00}\left(k^{0}=\omega_{p, n}^{f}\right)}{\left(\omega_{p, n}^{f}\right)^{2}-\left(\omega_{k, l}^{f}\right)^{2}}\right\}\right]+\operatorname{Re} \Pi_{\mathrm{vac}}^{00}\left(q_{z}\right)+\operatorname{Re} \Pi_{\mathrm{B}}^{00}\left(q_{z}, B\right),
\end{aligned}
$$

where

$$
\tilde{N}_{f, n l}^{00}=\left(\frac{\left|e_{f} B\right|}{\pi}\right)\left[4\left|e_{f} B\right| n \delta_{n-1}^{l-1}+\left(\delta_{n}^{l}+\delta_{n-1}^{l-1}\right)\left(m_{f}^{2}+k_{0}^{2}+k_{z}^{2}+k_{z} q_{z}\right)\right]
$$

The presence of the Kronecker delta function in the above equation will make the double sum of Eq. (B3) into a single sum. Finally taking the $q_{z} \rightarrow 0$ limit of Eq. (B3) we arrive at Eq. (20). It is worth mentioning that the last two terms of Eq. (B3) go to zero as $q_{z} \rightarrow 0$ so that they do not contribute in the Debye mass.

\section{APPENDIX C: SIMPLIFICATION OF THE SPECTRAL FUNCTION FOR $B \neq 0$ CASE}

In view of simplifications in analytic calculations, we choose the transverse momentum of the photon $q_{\perp}=0$ so that for $B \neq 0$, the spectral function $\rho\left(q^{0}, q_{z}\right)$ becomes [using Eqs. (21) and (34)]

$$
\begin{aligned}
\rho^{q \bar{q}}\left(q^{0}, q_{z}\right)= & \frac{1}{4 \pi \alpha} \operatorname{sign}\left(q^{0}\right) \tanh \left(\frac{\beta q^{0}}{2}\right) \sum_{f} e_{f}^{2} N_{c} \sum_{l=0}^{\infty} \sum_{n=0}^{\infty} \pi \int \frac{d^{3} k}{(2 \pi)^{3}} \frac{1}{4 \omega_{k, l}^{f} \omega_{p, n}^{f}}\left[U_{1, n l}^{f} \delta\left(q^{0}-\omega_{k, l}^{f}-\omega_{p, n}^{f}\right)\right. \\
& \left.+U_{2, n l}^{f} \delta\left(q^{0}+\omega_{k, l}^{f}+\omega_{p, n}^{f}\right)+L_{1, n l}^{f} \delta\left(q^{0}+\omega_{k, l}^{f}-\omega_{p, n}^{f}\right)+L_{2, n l}^{f} \delta\left(q^{0}-\omega_{k, l}^{f}+\omega_{p, n}^{f}\right)\right],
\end{aligned}
$$

where

$$
\begin{gathered}
U_{1, n l}^{f}=\left\{1-f\left(\omega_{k, l}^{f}\right)-f\left(\omega_{p, n}^{f}\right)+2 f\left(\omega_{k, l}^{f}\right) f\left(\omega_{p, n}^{f}\right)\right\} g_{\mu \nu} N_{f, n l}^{\mu \nu}\left(k^{0}=-\omega_{k, l}^{f}\right), \\
U_{2, n l}^{f}=\left\{1-f\left(\omega_{k, l}^{f}\right)-f\left(\omega_{p, n}^{f}\right)+2 f\left(\omega_{k, l}^{f}\right) f\left(\omega_{p, n}^{f}\right)\right\} g_{\mu \nu} N_{f, n l}^{\mu \nu}\left(k^{0}=\omega_{k, l}^{f}\right), \\
L_{1, n l}^{f}=\left\{-f\left(\omega_{k, l}^{f}\right)-f\left(\omega_{p, n}^{f}\right)+2 f\left(\omega_{k, l}^{f}\right) f\left(\omega_{p, n}^{f}\right)\right\} g_{\mu \nu} N_{f, n l}^{\mu \nu}\left(k^{0}=\omega_{k, l}^{f}\right), \\
L_{2, n l}^{f}=\left\{-f\left(\omega_{k, l}^{f}\right)-f\left(\omega_{p, n}^{f}\right)+2 f\left(\omega_{k, l}^{f}\right) f\left(\omega_{p, n}^{f}\right)\right\} g_{\mu \nu} N_{f, n l}^{\mu \nu}\left(k^{0}=-\omega_{k, l}^{f}\right),
\end{gathered}
$$

in which

$$
\begin{aligned}
N_{f, n l}^{\mu \nu}\left(q_{\|}, k\right) g_{\mu \nu}= & (-1)^{n+l} e^{-\alpha_{k}-\alpha_{p}} 16\left[-8 L_{l-1}^{1}\left(2 \alpha_{k}\right) L_{n-1}^{1}\left(2 \alpha_{p}\right)\left(k_{\perp}^{2}+k_{\perp} \cdot q_{\perp}\right)\right. \\
& +\left\{L_{l}\left(2 \alpha_{k}\right) L_{n}\left(2 \alpha_{p}\right)+L_{l-1}\left(2 \alpha_{k}\right) L_{n-1}\left(2 \alpha_{p}\right)\right\} m_{f}^{2} \\
& \left.-\left\{L_{l}\left(2 \alpha_{k}\right) L_{n-1}\left(2 \alpha_{p}\right)+L_{l-1}\left(2 \alpha_{k}\right) L_{n}\left(2 \alpha_{p}\right)\right\}\left(m_{f}^{2}-k_{\|}^{2}-k_{\|} \cdot q_{\|}\right)\right] .
\end{aligned}
$$

It is now trivial to perform the $d^{2} k_{\perp}$ integral in Eq. (C1) using the orthogonality of generalized Laguerre polynomials (identities provided in Appendix A) so that the spectral function becomes

$$
\begin{aligned}
\rho^{q \bar{q}}\left(q^{0}, q_{z}\right)= & \frac{1}{4 \pi \alpha} \operatorname{sign}\left(q^{0}\right) \tanh \left(\frac{\beta q^{0}}{2}\right) \sum_{f} e_{f}^{2} N_{c} \sum_{n=0}^{\infty} \sum_{l=(n-1)}^{(n+1)} \pi \int_{-\infty}^{\infty} \frac{d k_{z}}{2 \pi} \frac{1}{4 \omega_{k, l}^{f} \omega_{p, n}^{f}}\left[\tilde{U}_{1, n l}^{f} \delta\left(q^{0}-\omega_{k, l}^{f}-\omega_{p, n}^{f}\right)\right. \\
& \left.+\tilde{U}_{2, n l}^{f} \delta\left(q^{0}+\omega_{k, l}^{f}+\omega_{p, n}^{f}\right)+\tilde{L}_{1, n l}^{f} \delta\left(q^{0}+\omega_{k, l}^{f}-\omega_{p, n}^{f}\right)+\tilde{L}_{2, n l}^{f} \delta\left(q^{0}-\omega_{k, l}^{f}+\omega_{p, n}^{f}\right)\right],
\end{aligned}
$$

where 


$$
\begin{gathered}
\tilde{U}_{1, n l}^{f}=\left\{1-f\left(\omega_{k, l}^{f}\right)-f\left(\omega_{p, n}^{f}\right)+2 f\left(\omega_{k, l}^{f}\right) f\left(\omega_{p, n}^{f}\right)\right\} N_{f, n l}\left(k^{0}=-\omega_{k, l}^{f}\right), \\
\tilde{U}_{2, n l}^{f}=\left\{1-f\left(\omega_{k, l}^{f}\right)-f\left(\omega_{p, n}^{f}\right)+2 f\left(\omega_{k, l}^{f}\right) f\left(\omega_{p, n}^{f}\right)\right\} N_{f, n l}\left(k^{0}=\omega_{k, l}^{f}\right), \\
\tilde{L}_{1, n l}^{f}=\left\{-f\left(\omega_{k, l}^{f}\right)-f\left(\omega_{p, n}^{f}\right)+2 f\left(\omega_{k, l}^{f}\right) f\left(\omega_{p, n}^{f}\right)\right\} N_{f, n l}\left(k^{0}=\omega_{k, l}^{f}\right), \\
\tilde{L}_{2, n l}^{f}=\left\{-f\left(\omega_{k, l}^{f}\right)-f\left(\omega_{p, n}^{f}\right)+2 f\left(\omega_{k, l}^{f}\right) f\left(\omega_{p, n}^{f}\right)\right\} N_{f, n l}\left(k^{0}=-\omega_{k, l}^{f}\right),
\end{gathered}
$$

in which

$$
N_{f, n l}\left(q_{\|}, k_{\|}\right)=(-1)^{n+l}\left(\frac{\left|e_{f} B\right|}{\pi}\right) 2\left[4\left|e_{f} B\right| n \delta_{n-1}^{l-1}+\left(\delta_{n}^{l}+\delta_{n-1}^{l-1}\right) m_{f}^{2}+\left(\delta_{n-1}^{l}+\delta_{n}^{l-1}\right)\left(k_{\|}^{2}+k_{\|} \cdot q_{\|}-m_{f}^{2}\right)\right] .
$$

It is to be understood that a Kronecker delta with $-v e$ index is zero, i.e., $\delta_{l}^{l}=0$ if $l<0$, which comes from the fact that $L_{-1}^{a}(z)=0$ in the Schwinger propagator. The presence of Kronecker delta functions in the above equation have made the infinite double sums of Eq. (C1) into a single one or in other words the sum over $l$ runs from $(n-1)$ to $(n+1)$.

\section{APPENDIX D: KINEMATIC DOMAINS OF SPECTRAL FUNCTION}

Let us first consider the zero magnetic field case. The expression for the imaginary part of the self-energy, given in Eq. (12), contains four Dirac delta functions, and they will be nonvanishing in certain kinematic regions. To see this, let us consider $\delta\left(q^{0} \mp \omega_{k}^{f} \mp \omega_{p}^{f}\right)=\delta\left(q^{0} \mp E\right)$ and $\delta\left(q^{0} \mp \omega_{k}^{f} \pm \omega_{p}^{f}\right)=\delta\left(q^{0} \mp E^{\prime}\right)$ where $E=\left(\omega_{k}^{f}+\omega_{p}^{f}\right)$ and $E^{\prime}=\left(\omega_{k}^{f}-\omega_{p}^{f}\right)$. Both the functions $E=E(q, \vec{k}, \cos \theta)$ and $E^{\prime}=E^{\prime}(q, \vec{k}, \cos \theta)$ are defined in the domains $0 \leq$ $|\vec{k}|<\infty$ and $|\cos \theta| \leq 1$ so that their codomains come out to be

$$
\sqrt{\vec{q}^{2}+4 m_{f}^{2}} \leq E<\infty \quad \text { and } \quad-|\vec{q}| \leq E^{\prime} \leq|\vec{q}|
$$

Therefore, $\delta\left(q^{0}-E\right), \delta\left(q^{0}+E\right)$, and $\delta\left(q^{0} \mp E^{\prime}\right)$ are nonvanishing in the kinematic regions defined in terms of $\sqrt{\vec{q}^{2}+4 m_{f}^{2}} \leq q^{0}<\infty,-\infty<q^{0} \leq-\sqrt{\vec{q}^{2}+4 m_{f}^{2}}$, and $\left|q^{0}\right| \leq|\vec{q}|$, respectively.

Let us now turn on the external magnetic field. Analogous to the zero magnetic field case, the simplified spectral function in Eq. (C7) contains four Dirac delta functions, namely $\delta\left(q^{0} \mp \omega_{k, l}^{f} \mp \omega_{p, n}^{f}\right)=\delta\left(q^{0} \mp E_{n l}^{f}\right)$ and $\delta\left(q^{0} \mp \omega_{k, l}^{f} \pm \omega_{p, n}^{f}\right)=\delta\left(q^{0} \mp E_{n l}^{f}\right)$ where $E_{n l}^{f}=\omega_{k, l}^{f}+\omega_{p, n}^{f}$ and $E_{n l}^{\prime f}=\omega_{k, l}^{f}-\omega_{p, n}^{f}$. Both the functions $E_{n l}^{f}=$ $E_{n l}^{f}\left(q^{0}, q_{z}, k_{z}\right)$ and $E_{n l}^{\prime f}=E_{n l}^{\prime f}\left(q^{0}, q_{z}, k_{z}\right)$ are defined in the domain $-\infty<k_{z}<\infty$, and their codomains are given by

$$
\begin{gathered}
\sqrt{q_{z}^{2}+\left(m_{f, l}+m_{f, n}\right)^{2}} \leq E<\infty \quad \text { and } \\
\min \left(q_{z}, E_{ \pm}^{\prime}\right) \leq E^{\prime} \leq \max \left(q_{z}, E_{ \pm}^{\prime}\right)
\end{gathered}
$$

where $E_{ \pm}^{\prime}=\frac{m_{f, l}-m_{f, n}}{\left|m_{f, l} \pm m_{f, n}\right|} \sqrt{q_{z}^{2}+\left(m_{f, l} \pm m_{f, n}\right)^{2}}$. The above equation implies that, for a particular set $\{n, l\}$, $\delta\left(q^{0}-\omega_{k, l}^{f}-\omega_{p, n}^{f}\right)$ and $\delta\left(q^{0}+\omega_{k, l}^{f}+\omega_{p, n}^{f}\right)$ will be nonvanishing in the kinematic regions $\sqrt{q_{z}^{2}+\left(m_{f, l}+m_{f, n}\right)^{2}} \leq$ $q^{0}<\infty$ and $-\infty<q^{0} \leq-\sqrt{q_{z}^{2}+\left(m_{f, l}+m_{f, n}\right)^{2}}$, respectively. Whereas for $\delta\left(q^{0}-\omega_{k, l}^{f}+\omega_{p, n}^{f}\right)$ and $\delta\left(q^{0}+\omega_{k, l}^{f}-\omega_{p, n}^{f}\right)$, the corresponding kinematic regions will be, respectively, $\min \left(q_{z}, E_{ \pm}^{\prime}\right) \leq q^{0} \leq \max \left(q_{z}, E_{ \pm}^{\prime}\right) \quad$ and $\quad-\max \left(q_{z}, E_{ \pm}^{\prime}\right) \leq$ $q^{0} \leq-\min \left(q_{z}, E_{ \pm}^{\prime}\right)$. Therefore, in Eq. (C7), when the index $n$ is summed over from 0 to $\infty, \delta\left(q^{0}-\omega_{k, l}^{f}-\omega_{p, n}^{f}\right)$ and $\delta\left(q^{0}+\omega_{k, l}^{f}+\omega_{p, n}^{f}\right)$ will be nonvanishing at $\sqrt{q_{z}^{2}+4 m_{f}^{2}} \leq$ $q^{0}<\infty$ and $-\infty<q^{0} \leq \sqrt{q_{z}^{2}+4 m_{f}^{2}}$, respectively (for $n=0, l=0$ ). Corresponding regions for the nonvanishing of $\delta\left(q^{0} \mp \omega_{k, l}^{f} \pm \omega_{p, n}^{f}\right)$ will be $\left|q^{0}\right| \leq$ $\sqrt{q_{z}^{2}+\left(m_{f}-\sqrt{m_{f}^{2}+2\left|e_{f} B\right|}\right)^{2}}$ (for $\left.n=1, l=0\right)$. Note that the thresholds of the Unitary cuts arise when both of the two quarks in the loop lie in LLL. In contrast, the Landau cut threshold arises when one of the quarks in the loop is at LLL and the other one is at the next to LLL.

\section{APPENDIX E: CALCULATION OF $\boldsymbol{W}_{\boldsymbol{\mu}}^{\mathbf{1 1}}(\boldsymbol{q})$}

We have the expression of $W_{\mu \nu}^{11}(q)$ from Eq. (65) as

$$
W_{\mu \nu}^{11}(q)=i \int d^{4} x e^{i q \cdot x}\left\langle\mathcal{T} J_{\mu}(x) J_{\nu}^{\dagger}(0)\right\rangle .
$$

Substituting the quark/antiquark current $J^{\mu}(x)$ from Eq. (48) into the above equation, we get 
$W_{\mu \nu}^{11}(q)=i \int d^{4} x e^{i q \cdot x} \sum_{f} e_{f}^{2}\left\langle\mathcal{T} \bar{q}_{f}(x) \gamma_{\mu} q_{f}(x) \bar{q}_{f}(0) \gamma_{\nu} q_{f}(0)\right\rangle$.

Applying Wick's theorem [83], we get after some simplifications

$W_{\mu \nu}^{11}(q)=-i N_{c} \sum_{f} e_{f}^{2} \int d^{4} x e^{i q \cdot x} \operatorname{Tr}\left[\gamma_{\mu} S_{f}^{11}(x) \gamma_{\nu} S_{f}^{11}(-x)\right]$,

where $S_{f}^{11}(x)$ is the 11 component of the real time thermal quark propagator in coordinate space and can be Fourier transformed as

$S_{f}^{11}(x)=\left\langle\mathcal{T} q_{f}(x) \bar{q}_{f}(0)\right\rangle=\int \frac{d^{4} p}{(2 \pi)^{4}} e^{-i p \cdot x}\left(-i S_{f}^{11}(p)\right)$

in which $S_{f}^{11}(p)$ is defined in Eq. (7). Substituting the quark propagator into Eq. (E3), we get

$$
\begin{aligned}
W_{\mu \nu}^{11}(q)= & -i N_{c} \sum_{f} e_{f}^{2} \int d^{4} x e^{i q \cdot x} \int \frac{d^{4} k_{1}}{(2 \pi)^{4}} \\
& \times \int \frac{d^{4} k_{2}}{(2 \pi)^{4}} e^{-i x \cdot\left(k_{1}-k_{2}\right)}(-) \operatorname{Tr}\left[\gamma_{\mu} S_{f}^{11}\left(k_{1}\right) \gamma_{\nu} S_{f}^{11}\left(k_{2}\right)\right] .
\end{aligned}
$$

It is now trivial to perform the $d^{4} x$ integral giving a Dirac delta function $\delta^{4}\left(q-k_{1}+k_{2}\right)$, which in turn is used to perform one of the $d^{4} k$ integral. Finally we get

$$
W_{\mu \nu}^{11}(q)=N_{c} \sum_{f} e_{f}^{2} i \int \frac{d^{4} k}{(2 \pi)^{4}} \operatorname{Tr}\left[\gamma_{\mu} S_{f}^{11}(q+k) \gamma_{\nu} S_{f}^{11}(k)\right] .
$$

\section{APPENDIX F: CALCULATION OF $L_{\mu \nu}(q)$}

We have the expression for $L_{\mu \nu}(q)$ from Eq. (72) as

$$
L_{\mu \nu}(q)=i \int d^{4} x e^{i q \cdot x}\left\langle 0\left|\mathcal{T} j_{\nu}^{\dagger}(x) j_{\mu}(0)\right| 0\right\rangle .
$$

Substituting the lepton current $j^{\mu}(x)$ from Eq. (47) into the above equation, we get

$$
L_{\mu \nu}(q)=i \int d^{4} x e^{i q \cdot x} e^{2}\left\langle 0\left|\mathcal{T} \bar{\psi}(x) \gamma_{\nu} \psi(x) \bar{\psi}(0) \gamma_{\mu} \psi(0)\right| 0\right\rangle
$$

Applying Wick's theorem [83], we get after some simplifications

$$
L_{\mu \nu}(q)=-i e^{2} \int d^{4} x e^{i q \cdot x} \operatorname{Tr}\left[\gamma_{\nu} S_{l}(x) \gamma_{\mu} S_{l}(-x)\right]
$$

where $S_{l}(x)$ is the vacuum lepton propagator in coordinate space and can be Fourier transformed as

$$
S_{l}(x)=\langle 0|\mathcal{T} \psi(x) \bar{\psi}(0)| 0\rangle=\int \frac{d^{4} p}{(2 \pi)^{4}} e^{-i p \cdot x}\left(-i S_{l}(p)\right)
$$

in which $S_{l}(p)=\frac{-(\not+m)}{p^{2}-m^{2}+i \epsilon}$ is the corresponding vacuum momentum space lepton propagator. Substituting the lepton propagator into Eq. (F3), we get

$$
L_{\mu \nu}(q)=-i e^{2} \int d^{4} x e^{i q \cdot x} \int \frac{d^{4} k_{1}}{(2 \pi)^{4}} \int \frac{d^{4} k_{2}}{(2 \pi)^{4}} e^{-i x \cdot\left(k_{1}-k_{2}\right)}(-) \operatorname{Tr}\left[\gamma_{\nu} S_{l}\left(k_{1}\right) \gamma_{\mu} S_{l}\left(k_{2}\right)\right] .
$$

It is now trivial to perform the $d^{4} x$ integral giving a Dirac delta function $\delta^{4}\left(q-k_{1}+k_{2}\right)$, which in turn is used to perform one of the $d^{4} k$ integral. Finally we get

$$
L_{\mu \nu}(q)=e^{2} i \int \frac{d^{4} k}{(2 \pi)^{4}} \operatorname{Tr}\left[\gamma_{\nu} S_{l}(q+k) \gamma_{\mu} S_{l}(k)\right]
$$

\section{APPENDIX G: CALCULATION OF $L_{\mu \nu}^{+}$IN TWO DIFFERENT APPROACHES}

In this Appendix we will show that both Eqs. (62) and (71) give identical analytic expressions of $L_{\mu \nu}^{+}(q)$. To see this, let us start with Eq. (62),

$$
L_{\mu \nu}^{+}(q)=(2 \pi)^{4} \sum_{s_{+}, s_{-}} \int \frac{d^{3} p_{+}}{(2 \pi)^{3} 2 E_{+}} \int \frac{d^{3} p_{-}}{(2 \pi)^{3} 2 E_{-}} \delta^{4}\left(q-p_{+}-p_{-}\right)\left\langle l^{+} l^{-}\left|j_{\mu}(0)\right| 0\right\rangle\left\langle 0\left|j_{\nu}^{\dagger}(0)\right| l^{+} l^{-}\right\rangle .
$$


We now substitute lepton current $j^{\mu}(x)$ from Eq. (47) into the above equation to get

$$
L_{\mu \nu}^{+}(q)=(2 \pi)^{4} e^{2} \sum_{s_{+}, s_{-}} \int \frac{d^{3} p_{+}}{(2 \pi)^{3} 2 E_{+}} \int \frac{d^{3} p_{-}}{(2 \pi)^{3} 2 E_{-}} \delta^{4}\left(q-p_{+}-p_{-}\right)\left\langle l^{+} l^{-}\left|\bar{\psi}(0) \gamma_{\mu} \psi(0)\right| 0\right\rangle\left\langle 0\left|\bar{\psi}(0) \gamma_{\nu} \psi(0)\right| l^{+} l^{-}\right\rangle .
$$

In order to calculate the matrix elements in the above equation, we use the Fourier decompositions of the lepton fields in terms of creation/annihilation operators as [56]

$$
\begin{aligned}
& \psi(x)=\sum_{s} \int \frac{d^{3} p}{(2 \pi)^{3} 2 E_{p}}\left[u(s, p) e^{-i p \cdot x} a(s, p)+v(s, p) e^{i p \cdot x} b^{\dagger}(s, p)\right], \\
& \bar{\psi}(x)=\sum_{s} \int \frac{d^{3} p}{(2 \pi)^{3} 2 E_{p}}\left[\bar{v}(s, p) e^{-i p \cdot x} b(s, p)+\bar{u}(s, p) e^{i p \cdot x} a^{\dagger}(s, p)\right],
\end{aligned}
$$

so that the matrix elements come out to be

$$
\begin{gathered}
\left\langle l^{+} l^{-}\left|\bar{\psi}(0) \gamma_{\mu} \psi(0)\right| 0\right\rangle=\bar{u}\left(s_{-}, p_{-}\right) \gamma_{\mu} v\left(s_{+}, p_{+}\right), \\
\left\langle 0\left|\bar{\psi}(0) \gamma_{\nu} \psi(0)\right| l^{+} l^{-}\right\rangle=\left[\bar{u}\left(s_{-}, p_{-}\right) \gamma_{\nu} v\left(s_{+}, p_{+}\right)\right]^{*} .
\end{gathered}
$$

Substituting the matrix elements into Eq. (G2), we get

$$
L_{\mu \nu}^{+}(q)=(2 \pi)^{4} e^{2} \sum_{s_{+}, s_{-}} \int \frac{d^{3} p_{+}}{(2 \pi)^{3} 2 E_{+}} \int \frac{d^{3} p_{-}}{(2 \pi)^{3} 2 E_{-}} \delta^{4}\left(q-p_{+}-p_{-}\right) \bar{u}\left(s_{-}, p_{-}\right) \gamma_{\mu} v\left(s_{+}, p_{+}\right)\left[\bar{u}\left(s_{-}, p_{-}\right) \gamma_{\nu} v\left(s_{+}, p_{+}\right)\right]^{*} .
$$

Performing the spin sum over the lepton spinors, we get

$$
L_{\mu \nu}^{+}(q)=(2 \pi)^{4} e^{2} \int \frac{d^{3} p_{+}}{(2 \pi)^{3} 2 E_{+}} \int \frac{d^{3} p_{-}}{(2 \pi)^{3} 2 E_{-}} \delta^{4}\left(q-p_{+}-p_{-}\right) \operatorname{Tr}\left[\left(\not p_{-}+m\right) \gamma_{\mu}\left(\not p_{+}-m\right) \gamma_{\nu}\right] .
$$

Calculating the trace over Dirac matrices, and performing the $d^{3} p_{+}$integral using the Dirac delta function, we get

$$
L^{+\mu \nu}(q)=\left.8 \pi e^{2} \int \frac{d^{3} p_{-}}{(2 \pi)^{3}} \frac{1}{4 E_{+} E_{-}} \delta\left(q^{0}-E_{+}-E_{-}\right)\left[-g^{\mu \nu}\left(m^{2}-p_{-}^{2}+p_{-} \cdot q\right)+\left(q^{\mu} p_{-}^{\nu}+q^{\nu} p_{-}^{\mu}\right)-2 p_{-}^{\mu} p_{-}^{\nu}\right]\right|_{\vec{p}_{+}=\vec{q}-\vec{p}_{-}} .
$$

Let us now consider Eq. (71), which can be written using Eq. (73) as

$$
L_{\mu \nu}^{+}(q)=-2 \operatorname{Im} \pi^{\mu \nu}(q),
$$

where $\pi^{\mu \nu}(q)$ is the one-loop vacuum self-energy of the photon for a $l^{+} l^{-}$loop. We have already calculated the one-loop photon self-energy for the $q \bar{q}$ loop in Sec. III. Thus $\pi^{\mu \nu}$ is obtained from Eq. (12) by replacing $T \rightarrow 0, N_{c} \rightarrow 1, \sum_{f} e_{f}^{2} \rightarrow e^{2}$, and $m_{f} \rightarrow m$ so that we get

$$
\operatorname{Im} \pi^{\mu \nu}=\left.e^{2} \pi \int \frac{d^{3} k}{(2 \pi)^{3}} \frac{1}{4 E_{k} E_{p}} \delta\left(q^{0}-E_{k}-E_{p}\right) N^{\mu \nu}\left(k^{0}=-E_{k}\right)\right|_{\vec{p}=\vec{q}+\vec{k}},
$$

where $N^{\mu \nu}(q)$ can be read from Eq. (13) as

$$
N^{\mu \nu}(q, k)=\operatorname{Tr}\left[\gamma^{\nu}(\not q+\not k+m) \gamma^{\mu}(\not k+m)\right]=4\left[\left(m^{2}-k^{2}-k \cdot q\right) g^{\mu \nu}+2 k^{\mu} k^{\nu}+\left(k^{\mu} q^{\nu}+k^{\nu} q^{\mu}\right)\right] .
$$

It is worth mentioning that we have considered only the physical kinematic region $q^{2}>0, q^{0}>0$ while writing Eq. (G10) so that the Unitary-II cut does not contribute. It is now trivial to check that after a change of variable $\vec{k} \rightarrow-\vec{k}$ in Eq. (G10) followed by substitution into Eq. (G9) leads to the same expression of $L_{\mu \nu}^{+}(q)$ as obtained earlier in Eq. (G8). 
[1] J. Adams et al. (STAR Collaboration), Nucl. Phys. A757, 102 (2005).

[2] K. Adcox et al. (PHENIX Collaboration), Nucl. Phys. A757, 184 (2005).

[3] B. B. Back et al., Nucl. Phys. A757, 28 (2005).

[4] I. Arsene et al. (BRAHMS Collaboration), Nucl. Phys. A757, 1 (2005).

[5] L. Adamczyk et al. (STAR Collaboration), Nature (London) 548, 62 (2017).

[6] V. Skokov, A. Yu. Illarionov, and V. Toneev, Int. J. Mod. Phys. A 24, 5925 (2009).

[7] R. C. Duncan and C. Thompson, Astrophys. J. 392, L9 (1992).

[8] D. E. Kharzeev, K. Landsteiner, A. Schmitt, and H.-U. Yee, Lect. Notes Phys. 871, 1 (2013).

[9] D. Kharzeev and A. Zhitnitsky, Nucl. Phys. A797, 67 (2007).

[10] D. E. Kharzeev, L. D. McLerran, and H. J. Warringa, Nucl. Phys. A803, 227 (2008).

[11] K. Fukushima, D. E. Kharzeev, and H. J. Warringa, Phys. Rev. D 78, 074033 (2008).

[12] V. P. Gusynin, V. A. Miransky, and I. A. Shovkovy, Nucl. Phys. B462, 249 (1996).

[13] V. P. Gusynin, V. A. Miransky, and I. A. Shovkovy, Nucl. Phys. B563, 361 (1999).

[14] G. S. Bali, F. Bruckmann, G. Endrodi, Z. Fodor, S. D. Katz, S. Krieg, A. Schafer, and K. K. Szabo, J. High Energy Phys. 02 (2012) 044.

[15] M. N. Chernodub, Phys. Rev. D 82, 085011 (2010).

[16] M. N. Chernodub, Lect. Notes Phys. 871, 143 (2013).

[17] E. J. Ferrer, V. de la Incera, and C. Manuel, Phys. Rev. Lett. 95, 152002 (2005).

[18] E. J. Ferrer, V. de la Incera, and C. Manuel, Nucl. Phys. B747, 88 (2006).

[19] E. J. Ferrer and V. de la Incera, Phys. Rev. D 76, 045011 (2007).

[20] K. Fukushima and H. J. Warringa, Phys. Rev. Lett. 100, 032007 (2008).

[21] B. Feng, D. Hou, H.-c. Ren, and P.-p. Wu, Phys. Rev. Lett. 105, 042001 (2010).

[22] S. Fayazbakhsh and N. Sadooghi, Phys. Rev. D 82, 045010 (2010).

[23] S. Fayazbakhsh and N. Sadooghi, Phys. Rev. D 83, 025026 (2011).

[24] C. Y. Wong, Introduction to High-Energy Heavy Ion Collisions (World Scientific, Singapore, 1995).

[25] P. B. Arnold, G. D. Moore, and L. G. Yaffe, J. High Energy Phys. 12 (2001) 009.

[26] P. Aurenche, F. Gelis, and H. Zaraket, Phys. Rev. D 61, 116001 (2000).

[27] P. Aurenche, F. Gelis, and H. Zaraket, Phys. Rev. D 62, 096012 (2000).

[28] R. Chatterjee, L. Bhattacharya, and D. K. Srivastava, arXiv:0901.3610.

[29] J. Alam, B. Sinha, and S. Raha, Phys. Rep. 273, 243 (1996).

[30] J. Alam, S. Sarkar, P. Roy, T. Hatsuda, and B. Sinha, Ann. Phys. (N.Y.) 286, 159 (2000).

[31] K. Kajantie, J. I. Kapusta, L. D. McLerran, and A. Mekjian, Phys. Rev. D 34, 2746 (1986).
[32] L. D. McLerran and T. Toimela, Phys. Rev. D 31, 545 (1985).

[33] R. Rapp and J. Wambach, Adv. Nucl. Phys. 25, 1 (2000).

[34] H. A. Weldon, Phys. Rev. D 42, 2384 (1990).

[35] R. Rapp and H. van Hees, in Quark-Gluon Plasma 4, edited by R. C. Hwa and X.-N. Wang (World Scientific, Singapore, 2010), pp. 111-206.

[36] T. Matsui and H. Satz, Phys. Lett. B 178, 416 (1986).

[37] X.-N. Wang and M. Gyulassy, Phys. Rev. Lett. 68, 1480 (1992).

[38] A. M. Poskanzer and S. A. Voloshin, Phys. Rev. C 58, 1671 (1998).

[39] S. A. Voloshin, A. M. Poskanzer, and R. Snellings, LandoltBornstein 23, 293 (2010).

[40] J.-Y. Ollitrault, Phys. Rev. D 46, 229 (1992).

[41] P. F. Kolb and U. W. Heinz, in Quark-Gluon Plasma 3, edited by R. C. Hwa and X.-N. Wang (World Scientific, Singapore, 2004), pp. 634-714.

[42] K. Aamodt et al. (ALICE Collaboration), Phys. Rev. Lett. 105, 252302 (2010).

[43] V. Chandra and V. Ravishankar, Phys. Rev. D 84, 074013 (2011).

[44] K. Hattori and K. Itakura, Ann. Phys. (Amsterdam) 330, 23 (2013).

[45] K. Hattori and K. Itakura, Ann. Phys. (Amsterdam) 334, 58 (2013).

[46] J. Chao, L. Yu, and M. Huang, Phys. Rev. D 90, 045033 (2014); 91, 029903(E) (2015).

[47] W.-y. Tsai, Phys. Rev. D 10, 2699 (1974).

[48] J. C. D’Olivo, J. F. Nieves, and S. Sahu, Phys. Rev. D 67, 025018 (2003).

[49] K. Tuchin, Phys. Rev. C 87, 024912 (2013).

[50] K. Tuchin, Phys. Rev. C 88, 024910 (2013).

[51] N. Sadooghi and F. Taghinavaz, Ann. Phys. (Amsterdam) 376, 218 (2017).

[52] K. A. Mamo, J. High Energy Phys. 08 (2013) 083.

[53] A. Bandyopadhyay, C. A. Islam, and M. G. Mustafa, Phys. Rev. D 94, 114034 (2016).

[54] A. Bandyopadhyay and S. Mallik, Phys. Rev. D 95, 074019 (2017).

[55] V. Chandra and V. Sreekanth, Phys. Rev. D 92, 094027 (2015).

[56] S. Mallik and S. Sarkar, Hadrons at Finite Temperature (Cambridge University Press, Cambridge, UK, 2016).

[57] M. Kurian and V. Chandra, Phys. Rev. D 96, 114026 (2017).

[58] S. Mitra and V. Chandra, Phys. Rev. D 97, 034032 (2018).

[59] V. Goloviznin and H. Satz, Z. Phys. C 57, 671 (1993).

[60] A. Peshier, B. Kampfer, O. P. Pavlenko, and G. Soff, Phys. Rev. D 54, 2399 (1996).

[61] M. D'Elia, A. Di Giacomo, and E. Meggiolaro, Phys. Lett. B 408, 315 (1997).

[62] M. D’Elia, A. Di Giacomo, and E. Meggiolaro, Phys. Rev. D 67, 114504 (2003).

[63] P. Castorina and M. Mannarelli, Phys. Lett. B 644, 336 (2007).

[64] P. Castorina and M. Mannarelli, Phys. Rev. C 75, 054901 (2007).

[65] A. Dumitru and R. D. Pisarski, Phys. Lett. B 525, 95 (2002). 
[66] K. Fukushima, Phys. Lett. B 591, 277 (2004).

[67] S. K. Ghosh, T. K. Mukherjee, M. G. Mustafa, and R. Ray, Phys. Rev. D 73, 114007 (2006).

[68] H. Abuki and K. Fukushima, Phys. Lett. B 676, 57 (2009).

[69] H.-M. Tsai and B. Muller, J. Phys. G 36, 075101 (2009).

[70] V. M. Bannur, Phys. Lett. B 647, 271 (2007).

[71] V. M. Bannur, Phys. Rev. C 75, 044905 (2007).

[72] V. M. Bannur, J. High Energy Phys. 09 (2007) 046.

[73] N. Su and K. Tywoniuk, Phys. Rev. Lett. 114, 161601 (2015).

[74] W. Florkowski, R. Ryblewski, N. Su, and K. Tywoniuk, Phys. Rev. C 94, 044904 (2016).

[75] W. Florkowski, R. Ryblewski, N. Su, and K. Tywoniuk, Acta Phys. Pol. B 47, 1833 (2016).

[76] A. Bandyopadhyay, N. Haque, M. G. Mustafa, and M. Strickland, Phys. Rev. D 93, 065004 (2016).

[77] M. Y. Jamal, S. Mitra, and V. Chandra, Phys. Rev. D 95, 094022 (2017).

[78] A. Bazavov et al., Phys. Rev. D 80, 014504 (2009).

[79] A. Bazavov et al. (HotQCD Collaboration), Phys. Rev. D 90, 094503 (2014).

[80] M. Kurian, S. Mitra, and V. Chandra, arXiv:1805.07313.

[81] M. Kurian and V. Chandra, Phys. Rev. D 97, 116008 (2018).

[82] M. L. Bellac, Thermal Field Theory, Cambridge Monographs on Mathematical Physics (Cambridge University Press, Cambridge, UK, 2011).

[83] M.E. Peskin and D. V. Schroeder, An Introduction to Quantum Field Theory (Addison-Wesley, Reading, MA, 1995).

[84] J. S. Schwinger, Phys. Rev. 82, 664 (1951).

[85] A. Ayala, A. Sanchez, G. Piccinelli, and S. Sahu, Phys. Rev. D 71, 023004 (2005).

[86] A. Mukherjee, S. Ghosh, M. Mandal, P. Roy, and S. Sarkar, Phys. Rev. D 96, 016024 (2017).
[87] S. Ghosh, A. Mukherjee, M. Mandal, S. Sarkar, and P. Roy, Phys. Rev. D 96, 116020 (2017).

[88] S. Ghosh, A. Mukherjee, M. Mandal, S. Sarkar, and P. Roy, Phys. Rev. D 94, 094043 (2016).

[89] J. Jankowski, D. Blaschke, and O. Kaczmarek, arXiv:1508 .05322 .

[90] K. Bhattacharya, arXiv:0705.4275.

[91] A. Schaefer and M. H. Thoma, Phys. Lett. B 451, 195 (1999).

[92] A. Peshier and M. H. Thoma, Phys. Rev. Lett. 84, 841 (2000).

[93] M. G. Mustafa, A. Schafer, and M. H. Thoma, Phys. Rev. C 61, 024902 (1999).

[94] C. Greiner, N. Haque, M. G. Mustafa, and M. H. Thoma, Phys. Rev. C 83, 014908 (2011).

[95] A. E. Dorokhov and W. Broniowski, Phys. Rev. D 65, 094007 (2002).

[96] C. D. Roberts and A. G. Williams, Prog. Part. Nucl. Phys. 33, 477 (1994).

[97] T. Schäfer and E. V. Shuryak, Rev. Mod. Phys. 70, 323 (1998).

[98] C. Bonati, M. D’Elia, M. Mariti, M. Mesiti, F. Negro, A. Rucci, and F. Sanfilippo, Phys. Rev. D 95, 074515 (2017).

[99] J. Alexandre, Phys. Rev. D 63, 073010 (2001).

[100] A. Bandyopadhyay, B. Karmakar, N. Haque, and M. G. Mustafa, arXiv:1702.02875.

[101] P. Chakraborty, arXiv:1711.04404.

[102] A. N. Gorban, Model Reduction and Coarse-Graining Approaches for Multiscale Phenomena (Springer, New York, 2006), p. 117.

[103] P. Ehrenfest and T. Ehrenfest, The Conceptual Foundations of the Statistical Approach in Mechanics (Dover, Mineola, NY, 2002). 